\title{
Certain aspects of holomorphic function theory on some genus-zero arithmetic groups
}

\author{
Jay Jorgenson, Lejla Smajlović and Holger Then
}

\begin{abstract}
There are a number of fundamental results in the study of holomorphic function theory associated to the discrete group $\operatorname{PSL}(2, \mathbb{Z})$, including the following statements: the ring of holomorphic modular forms is generated by the holomorphic Eisenstein series of weights four and six, denoted by $E_{4}$ and $E_{6}$; the smallest-weight cusp form $\Delta$ has weight twelve and can be written as a polynomial in $E_{4}$ and $E_{6}$; and the Hauptmodul $j$ can be written as a multiple of $E_{4}^{3}$ divided by $\Delta$. The goal of the present article is to seek generalizations of these results to some other genus-zero arithmetic groups $\Gamma_{0}(N)^{+}$with square-free level $N$, which are related to 'Monstrous moonshine conjectures'. Certain aspects of our results are generated from extensive computer analysis; as a result, many of the space-consuming results are made available on a publicly accessible web site. However, we do present in this article specific results for certain low-level groups.
\end{abstract}

\section{Introduction and statement of results}

Consider the discrete group $\operatorname{PSL}(2, \mathbb{Z})$ which acts on the upper half plane $\mathbb{H}$. The quotient space $\operatorname{PSL}(2, \mathbb{Z}) \backslash \mathbb{H}$ has one cusp, which can be taken to be at $i \infty$. Let $\Gamma_{\infty}$ denote the stabilizer subgroup for the cusp at $i \infty$, which consists of isometries

$$
\left(\begin{array}{ll}
a & b \\
c & d
\end{array}\right) \in \operatorname{PSL}(2, \mathbb{Z})
$$

with $c=0$. For every integer $k \geqslant 2$, the holomorphic Eisenstein series $E_{2 k}(z)$ is defined by the absolutely convergent sum

$$
E_{2 k}(z):=\sum_{\gamma \in \Gamma_{\infty} \backslash \operatorname{PSL}(2, \mathbb{Z})}(c z+d)^{-2 k}, \quad \text { where } \gamma=\left(\begin{array}{ll}
* & * \\
c & d
\end{array}\right) .
$$

There is an abundance of important and classical formulae which can be wound back to the holomorphic Eisenstein series $E_{2 k}$. For example, if one defines

$$
G_{2 k}(z):=\sum_{(n, m) \in \mathbf{Z}^{2} \backslash\{(0,0)\}}(n z+m)^{-2 k},
$$

then $E_{2 k}(z)=G_{2 k}(z) / 2 \zeta(2 k)$, where $\zeta(s)$ is the Riemann zeta function. If we set $g_{2}=60 G_{4}$ and $g_{3}=140 G_{6}$, the modular discriminant

$$
\Delta(z):=e^{2 \pi i z} \prod_{n=1}^{\infty}\left(1-e^{2 \pi i n z}\right)^{24}
$$

Received 22 May 2015; revised 10 February 2016.

2010 Mathematics Subject Classification 11F12 (primary), 11F20 (secondary).

J. J. acknowledges grant support from NSF and PSC-CUNY grants, and H. T. acknowledges support from EPSRC grant EP/H005188/1. 
can be written as

$$
\Delta(z)=g_{2}^{3}(z)-27 g_{3}^{2}(z)=\frac{1}{1728}\left(E_{4}^{3}(z)-E_{6}^{2}(z)\right) .
$$

The function $\Delta$ is a weight-twelve cusp form with respect to $\operatorname{PSL}(2, \mathbb{Z})$, meaning that it vanishes as $z$ approaches $i \infty$. It can be shown that no smaller weight cusp form exists. Furthermore, $\Delta$ is related to the algebraic discriminant of the cubic equation $y^{2}=4 x^{3}-g_{2} x-g_{3}$, in the complex projective coordinates $[x, y, 1]$, which defines an elliptic curve associated to the modular parameter $z$.

All higher weight modular forms associated to $\operatorname{PSL}(2, \mathbb{Z})$, including Eisenstein series, can be written in terms of $E_{4}(z)$ and $E_{6}(z)$. For example, the formulae $E_{8}(z)=E_{4}^{2}(z), E_{10}(z)=$ $E_{4}(z) E_{6}(z)$, and

$$
691 E_{12}(z)=441 E_{4}(z)^{3}+250 E_{6}(z)^{2}
$$

are just the beginning of the never ending list of interesting relations which one can write.

Whereas the content of the above discussion is classical, there is a very modern component. The function

$$
j(z)=\frac{1728 E_{4}^{3}(z)}{E_{4}^{3}(z)-E_{6}^{2}(z)}
$$

is a weight-zero modular form on $\operatorname{PSL}(2, \mathbb{Z}) \backslash \mathbb{H}$, which can be viewed as the biholomorphic function that maps the one-point compactification of $\operatorname{PSL}(2, \mathbb{Z}) \backslash \mathbb{H}$ onto the Riemann sphere $\mathbb{P}^{1}$. If we set $q=e^{2 \pi i z}$, then one can expand $j(z)$ as a function of $q$, namely one has

$$
j(z)=\frac{1}{q}+744+196884 q+21493760 q^{2}+O\left(q^{3}\right) \quad \text { as } q \rightarrow 0 .
$$

In the 1970s, the coefficients in (3) were observed to be related to the sizes of the irreducible representations of the largest sporadic simple group, which is now known as 'the monster'. The observations were made precise through the 'Monstrous moonshine conjectures', some of which are proven in the celebrated work by Borcherds. We refer the interested reader to $[\mathbf{4}, \mathbf{5}]$ for a thorough account of the underlying mathematics and physics surrounding the moonshine conjectures as well as the mathematical history associated to $j(z)$.

Setting to the side the important formulae themselves, one can summarize the above discussion as the three following points. First, the ring of holomorphic modular forms associated to $\operatorname{PSL}(2, \mathbb{Z})$ is generated by $E_{4}$ and $E_{6}$. Second, the smallest-weight cusp form $\Delta$ has weight twelve and hence can be written as a polynomial in $E_{4}$ and $E_{6}$. Third, the Hauptmodul $j$ is equal to a multiple of $E_{4}^{3}$ divided by $\Delta$ and hence is a rational function in $E_{4}$ and $E_{6}$.

The goal of this article is to seek generalizations of the above three statements to certain other arithmetic groups related to the 'Monstrous moonshine conjectures'. Specifically, for any square-free positive integer $N$, let

$$
\begin{array}{r}
\Gamma_{0}(N)^{+}=\left\{e^{-1 / 2}\left(\begin{array}{ll}
a & b \\
c & d
\end{array}\right) \in \mathrm{SL}(2, \mathbb{R}): a d-b c=e, a, b, c, d, e \in \mathbb{Z}\right. \\
e|N, e| a, e|d, N| c\}
\end{array}
$$

and let $\overline{\Gamma_{0}(N)^{+}}=\Gamma_{0}(N)^{+} /\{ \pm \mathrm{Id}\}$, where Id denotes the identity matrix. Observe that $\operatorname{PSL}(2, \mathbb{Z})=\overline{\Gamma_{0}(1)^{+}}$. It has been shown that there are 43 square-free integers $N>1$ such that the quotient space $X_{N}:=\overline{\Gamma_{0}(N)^{+}} \backslash \mathbb{H}$ has genus zero (see [3]). Each group has one cusp, which we can always choose to be at $i \infty$. As stated in the title, the aim of this paper is to present results in the study of the holomorphic function theory associated to these 43 spaces. 
Let

$$
\eta(z)=e^{2 \pi i z / 24} \prod_{n=1}^{\infty}\left(1-e^{2 \pi i n z}\right)
$$

denote the Dedekind eta function. For any square-free $N$, assume that $N$ has $r$ prime factors, and let $\operatorname{lcm}(\cdot, \cdot)$ denote the least common multiple function. Let $\sigma(N)$ equal the sum of divisors of $N$. It was proven in [7] that the function

$$
\Delta_{N}(z)=\left(\prod_{v \mid N} \eta(v z)\right)^{\ell_{N}},
$$

where

$$
\ell_{N}=2^{1-r} \operatorname{lcm}\left(4,2^{r-1} \frac{24}{(24, \sigma(N))}\right)
$$

is a weight- $k_{N}=2^{r-1} \ell_{N}$ modular form on $\overline{\Gamma_{0}(N)^{+}}$, vanishing at the cusp $i \infty$ only. For reasons discussed in [7], we refer to $\Delta_{N}$ as the Kronecker limit function on $\overline{\Gamma_{0}(N)^{+}}$.

The main results of the present paper are the following statements, which hold true for each square-free $N$ provided that $X_{N}$ has genus zero.

(1) There is an explicitly computed integer $M_{N}$ such that $\Delta_{N}^{M_{N}}$ is equal to a polynomial $Q_{N}$ in holomorphic Eisenstein series associated to $\overline{\Gamma_{0}(N)^{+}}$.

(2) The Hauptmodul $j_{N}$ associated to $\overline{\Gamma_{0}(N)^{+}}$is equal to a rational function whose numerator is a polynomial $P_{N}$ in holomorphic Eisenstein series and whose denominator is $\Delta_{N}^{M_{N}}$.

(3) The polynomials $P_{N}$ and $Q_{N}$ are explicitly computed; hence, we determine, for each $N$, a finite set $\mathcal{T}^{(N)}$ of holomorphic Eisenstein series such that any meromorphic form with at most polynomial growth at $i \infty$ can be expressed as a rational function involving elements of $\mathcal{T}^{(N)}$.

Points 1 and 2 are direct generalizations of the formulae (1) and (2). Point 3 is a weak generalization of the result that the ring of holomorphic modular forms associated to $\operatorname{PSL}(2, \mathbb{Z})$ is generated by the holomorphic Eisenstein series of weights four and six. For certain small levels, we are able to compute generators of the ring of holomorphic forms; however, for general $N$, and for future investigations we plan to undertake, we are content with point 3 as stated.

The present article is organized as follows. In $\S 2$, we establish notation and cite appropriate background material. In particular, we recall the Kronecker limit formula associated to the non-parabolic Eisenstein series on $X_{N}=\overline{\Gamma_{0}(N)^{+}} \backslash \mathbb{H}$ and a computer algorithm of [7]. In $\S 3$, we prove some basic results regarding low-weight modular forms for any level $N>1$. In $\S 4$, we present a variant of the algorithm of $[7]$ from which we prove that for every square-free $N$, provided that $X_{N}$ has genus zero, there is an integer $M_{N}$ such that $\Delta_{N}^{M_{N}}$ can be written as a polynomial in holomorphic Eisenstein series. Let $j_{N}$ denote the biholomorphic map from the one-point compactification of $X_{N}$ to the Riemann sphere $\mathbb{P}^{1}$ which maps $i \infty$ to zero. The algorithm described in $\S 4$ allows us to prove that $j_{N} \Delta_{N}^{M_{N}}$ can be written as a polynomial in holomorphic Eisenstein series. The data provided by the algorithm is presented in Table 1, as is a comparison of the results of the original algorithm of [7] and the modified variant thereof. From the algorithm developed in this paper, we are able to determine for each level $N$ a set of holomorphic Eisenstein series which generate $\mathcal{T}^{(N)}$, the ring of holomorphic modular forms associated to $X_{N}$; this information is given in Table 2. It is important to note that the entries in Table 2 may not be a minimal set of generators, meaning that for each $N$ there may exist further relations amongst the elements of sets listed in Table 2 . In $\S 5$, we present results regarding the ring of holomorphic forms for certain small levels.

As $N$ grows, so does the complexity of the formulae for $\Delta_{N}$ and $j_{N}$. For example, when $N=17$, our algorithm shows that the five holomorphic Eisenstein series of weights four 
through twelve generate $\mathcal{T}^{(17)}$ and $M_{17}=9$, meaning that $\Delta_{17}^{9}$ and $j_{17} \Delta_{17}^{9}$ can be written as a polynomial in these five Eisenstein series. As an indication of the complexity of the formulae, we present these two examples in $\S 5$. The formulae for $\Delta_{17}$ and $j_{17}$ each occupy approximately one page.

We note that the Tables 3 and 4a of [2] describe, in their notation, how one can express each Hauptmodul $j_{N}$ in terms of holomorphic forms. In Table 3, we translate the aforementioned data from [2], related to 43 groups defined by (4) with square-free $N$ and genus zero, such that we explicitly write these formulae in terms of the Dedekind eta function and theta function attached to quadratic forms. By combining our formulae for $j_{N}$ and the formulae from [2], one has the prospect of obtaining further identities involving holomorphic Eisenstein series and theta functions.

As in [7], the theoretical work developed in this article is supplemented by extensive computer analysis and, quite frankly, some of the results are not printable. For example, for $N=119$, the formula for $j_{119}$ from [7] occupies nearly 60 pages. Nonetheless, in order to disseminate the results obtained by our algorithms, we have posted all formulae to a web site [8].

\section{Background material}

\subsection{Holomorphic modular forms}

Let $\Gamma$ be a Fuchsian group of the first kind. Following [10], we define a weakly modular form $f$ of weight $2 k$ for $k \geqslant 1$ associated to $\Gamma$ to be a function $f$ which is meromorphic on $\mathbb{H}$ and satisfies the transformation property

$$
f\left(\frac{a z+b}{c z+d}\right)=(c z+d)^{2 k} f(z) \text { for all }\left(\begin{array}{ll}
a & b \\
c & d
\end{array}\right) \in \Gamma .
$$

Assume that $\Gamma$ has at least one class of parabolic elements. By transforming coordinates, if necessary, we may always assume that the parabolic subgroup of $\Gamma$ has a fixed point at $i \infty$, with identity scaling matrix. In this situation, any weakly modular form $f$ will satisfy the relation $f(z+1)=f(z)$, so we can write

$$
f(z)=\sum_{n=-\infty}^{\infty} a_{n} q^{n}, \quad \text { where } q=e^{2 \pi i z}
$$

If $a_{n}=0$ for all $n<0$, then $f$ is said to be holomorphic in the cusp $i \infty$. The form $f$ is said to vanish in the cusp $i \infty$ if $a_{n}=0$ for all $n \leqslant 0$. A holomorphic modular form with respect to $\Gamma$ is a weakly modular form which is holomorphic on $\mathbb{H}$ and is holomorphic in all of the cusps of $\Gamma$. A holomorphic cusp form is a holomorphic form which vanishes in all of the cusps of $\Gamma$.

For $\Gamma=\operatorname{PSL}(2, \mathbb{Z})$, the full modular surface, there is no weight-two holomorphic modular form. Nonetheless, one defines the function $E_{2}(z)$ by the $q$-expansion

$$
E_{2}(z)=1-24 \sum_{n=1}^{\infty} \sigma(n) q^{n} .
$$

It can be shown that $E_{2}(z)$ transforms according to the formula

$$
E_{2}(\gamma z)=(c z+d)^{2} E_{2}(z)+\frac{6}{\pi i} c(c z+d) \quad \text { for } \gamma=\left(\begin{array}{ll}
* & * \\
c & d
\end{array}\right) \in \operatorname{SL}(2, \mathbb{Z}) .
$$

From this, it is elementary to show that for a prime $p$, the function

$$
E_{2, p}(z):=\frac{p E_{2}(p z)-E_{2}(z)}{p-1}
$$


is a weight-two holomorphic form associated to the congruence subgroup $\Gamma_{0}(p)$ of $\operatorname{SL}(2, \mathbb{Z})$. The $q$-expansion of $E_{2, p}$ is

$$
E_{2, p}(z)=1+\frac{24}{p-1} \sum_{n=1}^{\infty} \sigma(n)\left(q^{n}-p q^{p n}\right) .
$$

\subsection{Certain arithmetic groups related to 'moonshine'}

For any square-free integer $N$, the subset of $\operatorname{SL}(2, \mathbb{R})$ defined by (4) is an arithmetic subgroup of $\operatorname{SL}(2, \mathbb{R})$. As shown in [3], there are precisely 44 such groups which have genus zero and which appear in 'Monstrous moonshine conjectures'. In this article we will focus on the 43 genus-zero groups for which $N>1$.

We denote by $\overline{\Gamma_{0}(N)^{+}}=\Gamma_{0}(N)^{+} /\{ \pm \mathrm{Id}\}$ the corresponding subgroup of $\operatorname{PSL}(2, \mathbb{R})$. Basic properties of $\Gamma_{0}(N)^{+}$for square-free $N$ are derived in $[\mathbf{6}]$ and references therein. In particular, we use that the surface $X_{N}=\overline{\Gamma_{0}(N)^{+}} \backslash \mathbb{H}$ has exactly one cusp, which can be taken to be at $i \infty$.

Let $\mathfrak{T}^{(N)}$ denote the ring of holomorphic modular forms associated to $X_{N}$, and let $\mathcal{T}_{2 k}^{(N)}$ denote the holomorphic modular forms of weight $2 k$. We will denote the subspace of cusp forms on $X_{N}$ of weight $2 k$ by $S_{2 k}^{(N)}$.

\subsection{Holomorphic Eisenstein series on $\overline{\Gamma_{0}(N)^{+}}$}

In the case when $N>1$ is square-free, the holomorphic Eisenstein series associated to $\overline{\Gamma_{0}(N)^{+}}$ are defined for $k \geqslant 2$ by

$$
E_{2 k}^{(N)}(z):=\sum_{\gamma \in \Gamma_{\infty}(N) \backslash \Gamma_{0}(N)^{+}}(c z+d)^{-2 k} \quad \text { with } \gamma=\left(\begin{array}{ll}
* & * \\
c & d
\end{array}\right),
$$

where $\Gamma_{\infty}(N)$ denotes the stabilizer group of the cusp at $i \infty$. Note that for all $N, \Gamma_{\infty}(N)$ is independent of $N$, namely one has that $\Gamma_{\infty}(N)=\Gamma_{\infty}$. In [7], it is proven that $E_{2 k}^{(N)}(z)$ may be expressed as a linear combination of forms $E_{2 k}(z)$, the holomorphic Eisenstein series associated to $\operatorname{PSL}(2, \mathbb{Z})$. Namely, it is known that

$$
E_{2 k}^{(N)}(z)=\frac{1}{\sigma_{k}(N)} \sum_{v \mid N} v^{k} E_{2 k}(v z)
$$

where $\sigma_{\alpha}$ denotes the generalized divisor function

$$
\sigma_{\alpha}(m)=\sum_{\delta \mid m} \delta^{\alpha}
$$

Formula (7), together with a well-known $q$-expansion of classical forms $E_{2 k}$, yields that the $q$-expansion of $E_{2 k}^{(N)}$ is given by

$$
E_{2 k}^{(N)}(z)=\frac{1}{\sigma_{k}(N)} \sum_{v \mid N} v^{k}\left(1-\frac{4 k}{B_{2 k}} \sum_{j=1}^{\infty} \sigma_{2 k-1}(j) q^{v j}\right),
$$

where $B_{k}$ denotes the $k$ th Bernoulli number. 


\subsection{Kronecker limit function on $\overline{\Gamma_{0}(N)^{+}}$}

Associated to the cusp of $\overline{\Gamma_{0}(N)^{+}}$one has a non-holomorphic Eisenstein series denoted by $\mathcal{E}_{\infty}^{\mathrm{par}}(z, s)$, which is defined for $z \in \mathbb{H}$ and $\operatorname{Re}(s)>1$ by

$$
\mathcal{E}_{\infty}^{\mathrm{par}}(z, s)=\sum_{\eta \in \Gamma_{\infty} \backslash \Gamma_{0}(N)^{+}} \operatorname{Im}(\eta z)^{s} .
$$

In [7] it is proven that, for any square-free $N$ which has $r$ prime factors, the parabolic Eisenstein series $\mathcal{E}_{\infty}^{\text {par }}(z, s)$ admits a Taylor series expansion of the form

$$
\mathcal{E}_{\infty}^{\mathrm{par}}(z, s)=1+s \cdot \log \left(\sqrt[2 r]{\prod_{v \mid N}|\eta(v z)|^{4}} \cdot \operatorname{Im}(z)\right)+O\left(s^{2}\right) \quad \text { as } s \rightarrow 0,
$$

where $\eta(z)$ is Dedekind's eta function associated to $\operatorname{PSL}(2, \mathbb{Z})$. As stated above, it is proven that the function

$$
\Delta_{N}(z)=\left(\prod_{v \mid N} \eta(v z)\right)^{\ell_{N}}
$$

where

$$
\ell_{N}=2^{1-r} \operatorname{lcm}\left(4,2^{r-1} \frac{24}{(24, \sigma(N))}\right)
$$

and $\operatorname{lcm}(\cdot, \cdot)$ denotes the least common multiple function, is a weight- $k_{N}=2^{r-1} \ell_{N}$ modular form on $\overline{\Gamma_{0}(N)^{+}}$, vanishing at the cusp $i \infty$ only. We call the function $\Delta_{N}(z)$ defined by (9) the Kronecker limit function on $\overline{\Gamma_{0}(N)^{+}}$.

\subsection{The algorithm}

Let $X_{N}=\overline{\Gamma_{0}(N)^{+}} \backslash \mathbb{H}$ have genus $g$. For any positive integer $M$, the function

$$
F_{b}(z)=\prod_{\nu}\left(E_{m_{\nu}}^{(N)}(z)\right)^{b_{\nu}} /\left(\Delta_{N}(z)\right)^{M}, \quad \text { where } \sum_{\nu} b_{\nu} m_{\nu}=M k_{N} \quad \text { and } \quad b=\left(b_{1}, \ldots\right)
$$

is a holomorphic modular function on $X_{N}$, meaning a weight-zero modular form with polynomial growth near $i \infty$. The $q$-expansion of $F_{b}$ follows from substituting the $q$-expansions of $E_{k}^{(N)}$ and $\Delta_{N}$.

Let $\mathcal{S}_{M}$ denote the set of all possible rational functions defined in (10) for all vectors $b=\left(b_{\nu}\right)$ and $m=\left(m_{\nu}\right)$ with fixed $M$. In [7], we implemented the following algorithm, which we refer to as the JST2 algorithm.

Choose a non-negative integer $\kappa$. Let $M=1$ and set $\mathcal{S}=\mathcal{S}_{1} \cup \mathcal{S}_{0}$.

(1) Form the matrix $A_{\mathcal{S}}$ of coefficients from the $q$-expansions of all elements of $\mathcal{S}$, where each element in $\mathcal{S}$ is expanded along a row with each column containing the coefficient of a power, negative, zero, or positive, of $q$. The expansion is recorded out to order $q^{\kappa}$.

(2) Apply Gauss elimination to $A_{\mathcal{S}}$, thus producing a matrix $B_{\mathcal{S}}$ which is in row-reduced echelon form.

(3) Implement the following decision to determine if the algorithm is complete: if the $g+1$ lowest non-trivial rows at the bottom of $B_{\mathcal{S}}$ correspond to $q$-expansions whose leading terms have precisely $g$ gaps, meaning zero coefficients, in the set $\left\{q^{-1}, \ldots, q^{-2 g}\right\}$, then the algorithm is completed. If the indicator to stop fails, then replace $M$ by $M+1, \mathcal{S}$ by $\mathcal{S}_{M} \cup \mathcal{S}$, and reiterate the algorithm. 
If $g=0$, then the algorithm stops if the lowest non-trivial row at the bottom of $B_{\mathfrak{S}}$ has a $q$-expansion which begins with $q^{-1}$. We also denote by $M_{N}$ the value of $M$ for the group of level $N$ at which Step (3) shows that the algorithm is completed.

As stated in [7], the rationale for the stopping decision in Step (3) above is based on two ideas, one factual and one hopeful. First, the Weierstrass gap theorem states that for any point $P$ on a compact Riemann surface there are precisely $g$ gaps in the set of possible orders from 1 to $2 g$ of functions whose only pole is at $P$. Second, for any genus, the assumption which is hopeful is that the function field is generated by the set of holomorphic modular functions defined in (10), which is related to the question of whether the field of meromorphic modular forms on $\overline{\Gamma_{0}(N)^{+}}$is generated by holomorphic Eisenstein series and the Kronecker limit function. The latter assumption is not obvious and, indeed, the assumption itself is equivalent to the statement that the rational function field on $X_{N}$ is generated by the holomorphic Eisenstein series. As it turned out, for all groups $\overline{\Gamma_{0}(N)^{+}}$that we have studied so far, which includes all groups of genus zero, genus one, genus two, and genus three, the algorithm stopped. Therefore, we conclude that, in particular, the rational function field associated to all genus-zero groups $\overline{\Gamma_{0}(N)^{+}}$is generated by a finite set of holomorphic Eisenstein series.

We described the algorithm with choice of arbitrary $\kappa$ and $g$. For reasons of efficiency, we initially selected $\kappa$ to be zero, so that all coefficients for $q^{\nu}$ for $\nu \leqslant \kappa$ are included in $A_{\mathcal{S}}$. In [7], it is shown that for each $N$, there is an explicitly computable $\kappa=\kappa_{N}$ such that if a modular form associated to $\overline{\Gamma_{0}(N)^{+}}$has integral coefficients in its $q$-expansion out to $q^{\kappa_{N}}$, then all remaining coefficients are also integral. The list of $\kappa_{N}$ for square-free levels $N$ provided that $\overline{\Gamma_{0}(N)^{+}}$has genus zero is given in Table 1 of [7]. In the implementation of the above algorithm, both in the present article and in [7], the value of $\kappa$ was finally increased to $\kappa_{N}$.

In the present article, we implemented a slight variant of the above algorithm, which we refer to as the JST3 algorithm. The difference between the JST2 and the JST3 algorithm is the following action should the decision in Step (3) fail.

Replace $M$ by $M+1, \mathcal{S}$ by $\mathcal{S}_{M}$, and reiterate the algorithm.

In other words, the JST3 algorithm studies the $q$-expansions of the space of rational functions of the form (10) with a fixed denominator $\left(\Delta_{N}(z)\right)^{M}$. Should the JST3 algorithm successfully complete, then the row in $B_{\mathcal{S}}$ with $q$-expansion beginning with $q^{-1}$ would correspond to a formula for $j_{N}$ with denominator $\left(\Delta_{N}(z)\right)^{M}$ and numerator given as a polynomial in Eisenstein series. Furthermore, any lower row in $B_{\mathcal{S}}$ would correspond to a $q$-expansion beginning with $q^{0}$, which would yield, upon clearing the denominator, a formula for $\left(\Delta_{N}(z)\right)^{M}$ in terms of Eisenstein series.

As we will report below, the JST3 algorithm has successfully completed for all genus-zero groups $\overline{\Gamma_{0}(N)^{+}}$with square-free level $N$.

\section{Modular forms on surfaces $X_{N}$}

From [1, Proposition 7, p. II-7], we immediately obtain the following formula, which relates the number of zeros of a modular form, counted with multiplicity, with its weight and the volume of $X_{N}$.

Lemma 1. Let $f$ be a modular form on $X_{N}$ of weight $2 k$, not identically zero. Let $\mathcal{F}_{N}$ denote the fundamental domain of $\overline{\Gamma_{0}(N)^{+}}$and let $v_{z}(f)$ denote the order of zero $z$ of $f$. Then

$$
k \frac{\operatorname{Vol}\left(X_{N}\right)}{2 \pi}=v_{i \infty}(f)+\sum_{e \in \mathcal{E}_{N}} \frac{1}{n_{e}} v_{e}(f)+\sum_{z \in \mathcal{F}_{N} \backslash \varepsilon_{N}} v_{z}(f),
$$


where $\mathcal{E}_{N}$ denotes the set of elliptic points in $\mathcal{F}_{N}$ and $n_{e}$ is the order of the elliptic point $e \in \mathcal{E}_{N}$.

Lemma 1 enables us to deduce the following proposition.

Proposition 2. Let $N$ be a square-free number such that the surface $X_{N}$ has genus zero. Then there are no weight-two holomorphic modular forms on $X_{N}$.

Proof. From the tables in [3], one determines that all genus-zero groups $\overline{\Gamma_{0}(N)^{+}}$for a squarefree $N$ have at most one elliptic point of order three, four, or six and a various number of order-two elliptic points. Let $e_{N}(2)$ denote the number of order-two elliptic points on $X_{N}$, and let $n_{N} \in\{3,4,6\}$ denote the order of the additional elliptic point on $X_{N}$. Since all surfaces $X_{N}$ have one cusp and genus zero, the Gauss-Bonnet formula for the volume of the surface $X_{N}$ becomes

$$
\frac{\operatorname{Vol}\left(X_{N}\right)}{2 \pi}=\frac{1}{2} e_{N}(2)+\left(1-\frac{1}{n_{N}}\right) \delta(N)-1,
$$

where $\delta(N)$ is equal to 1 if $X_{N}$ has an elliptic point of order different from two, and zero otherwise.

For an arbitrary, square-free $N$ and $e \mid N$, the elliptic element of $\overline{\Gamma_{0}(N)^{+}}$is of the form

$$
\left(\begin{array}{cc}
a \sqrt{e} & b / \sqrt{e} \\
(c N) / \sqrt{e} & d \sqrt{e}
\end{array}\right)
$$

where $a, b, c, d \in \mathbb{Z}$ are such that $|(a+d) \sqrt{e}|<2$ and $a d e-(b c N) / e=1$. The first condition implies that either $a+d=0$ or $|a+d|=1$ and $e \in\{1,2,3\}$.

If $|a+d|=1$, then $d= \pm 1-a$; hence,

$$
\left(\begin{array}{cc}
a \sqrt{e} & b / \sqrt{e} \\
(c N) / \sqrt{e} & d \sqrt{e}
\end{array}\right)^{2}=\left(\begin{array}{cc} 
\pm a e-1 & \pm b \\
\pm c N & \pm a e-1
\end{array}\right) \neq \pm \mathrm{Id}
$$

for any choice of $a, b, c \in \mathbb{Z}$ such that $a( \pm 1-a) e-(b c N) / e=1$. Therefore, there are no order-two elements in $\overline{\Gamma_{0}(N)^{+}}$such that $|a+d|=1$.

On the other hand, if $a+d=0$, then $-a^{2} e-(b c N) / e=1$; hence,

$$
\left(\begin{array}{cc}
a \sqrt{e} & b / \sqrt{e} \\
(c N) / \sqrt{e} & d \sqrt{e}
\end{array}\right)^{2}=\left(\begin{array}{cc}
-1 & 0 \\
0 & -1
\end{array}\right)
$$

In other words, any elliptic element $(13)$ of $\overline{\Gamma_{0}(N)^{+}}$has order two if and only if $a+d=0$. Let

$$
\eta=\left(\begin{array}{cc}
a \sqrt{e} & b / \sqrt{e} \\
(c N) / \sqrt{e} & -a \sqrt{e}
\end{array}\right)
$$

denote an arbitrary elliptic element of $\overline{\Gamma_{0}(N)^{+}}$of order two, and let $z_{\eta}$ be its fixed point. Solving the equation $\eta\left(z_{\eta}\right)=z_{\eta}$ leads to the conclusion that $\left(z_{\eta} c N / \sqrt{e}-a \sqrt{e}\right)^{2}=-1$.

Assume that $f_{2, N}$ is a holomorphic modular form on $X_{N}$ of weight two. By the transformation rule, we have that

$$
f_{2, N}\left(z_{\eta}\right)=f_{2, N}\left(\eta z_{\eta}\right)=(-1) f_{2, N}\left(z_{\eta}\right)
$$

and hence $z_{\eta}$ is a vanishing point of $f_{2, N}$. Since this holds true for any order-two elliptic element of $\overline{\Gamma_{0}(N)^{+}}$, we conclude that all order-two elliptic points of $X_{N}$ are vanishing points of $f_{2, N}$. Applying Lemma 1 to $f_{2, N}$, we arrive at the inequality

$$
\frac{\operatorname{Vol}\left(X_{N}\right)}{2 \pi} \geqslant \frac{1}{2} e_{N}(2)
$$


which contradicts (12). Therefore, there are no weight-two holomorphic modular forms on $X_{N}$.

Though there are no weight-two holomorphic forms on $\Gamma_{0}(N)^{+}$, we may construct forms that transform almost like a weight-two form, up to an order-two character.

Proposition 3. Let $N=p_{1} \ldots p_{r}$ be a square-free positive integer with $N>1$. Let $\mu(\nu)$ denote the Möbius function and $E_{2}$ the series defined in (5). Then the holomorphic function

$$
E_{2, N}(z):=\frac{(-1)^{r}}{\varphi(N)} \sum_{v \mid N} \mu(v) v E_{2}(v z)
$$

satisfies the transformation rule

$$
E_{2, N}\left(\gamma_{e} z\right)=\mu(e)\left(c \frac{N}{\sqrt{e}} z+d \sqrt{e}\right)^{2} E_{2, N}(z)
$$

for any

$$
\gamma_{e}=\left(\begin{array}{cc}
a \sqrt{e} & b / \sqrt{e} \\
(c N) / \sqrt{e} & d \sqrt{e}
\end{array}\right) \in \Gamma_{0}(N)^{+}
$$

Proof. Choose and fix any $e \mid N$. For any $v \mid N$, let $(e, v)$ denote the greatest common divisor of $e$ and $v$. Then, using the transformation formula (6) for $E_{2}$, it is easy to deduce that

$$
v E_{2}\left(v\left(\gamma_{e} z\right)\right)=\frac{e v}{(e, v)^{2}}\left(c \frac{N}{\sqrt{e}} z+d \sqrt{e}\right)^{2} E_{2}\left(\frac{e v}{(e, v)^{2}} z\right)+\frac{6}{\pi i} c N\left(c \frac{N}{e} z+d\right) .
$$

Since $N$ is square-free with $r$ prime factors, it is easy to see that

$$
\sum_{v \mid N} \mu(v) \frac{6}{\pi i} c N\left(c \frac{N}{e} z+d\right)=\frac{6}{\pi i} c N\left(c \frac{N}{e} z+d\right) \sum_{j=1}^{r}\left(\begin{array}{l}
r \\
j
\end{array}\right)(-1)^{j}=0
$$

and hence

$$
\sum_{v \mid N} \mu(v) v E_{2}\left(v\left(\gamma_{e} z\right)\right)=\sum_{v \mid N} \mu(v) \frac{e v}{(e, v)^{2}}\left(c \frac{N}{\sqrt{e}} z+d \sqrt{e}\right)^{2} E_{2}\left(\frac{e v}{(e, v)^{2}} z\right) .
$$

We claim that $\{v: v \mid N\}=\left\{e v /(e, v)^{2}: v \mid N\right\}$, which is easily deduced by induction in $r$. Furthermore, when $e$ has an even number of prime factors, the parity of the number of factors of $e v /(e, v)^{2}$ remains the same as the parity of the number of factors of $v$, while when $e$ has an odd number of factors, the parity changes, meaning that $\mu(v)=\mu(e) \mu\left(e v /(e, v)^{2}\right)$. Therefore,

$$
\sum_{v \mid N} \mu(v) v E_{2}\left(v\left(\gamma_{e} z\right)\right)=\mu(e)\left(c \frac{N}{\sqrt{e}} z+d \sqrt{e}\right)^{2} \sum_{v \mid N} \mu(v) v E_{2}(v z)
$$

and the proof is complete.

Proposition 4. The smallest even integer $k_{N}$ such that there exists a weight- $k_{N}$ cusp form $f_{N}$ vanishing only at the cusp $i \infty$ is given by the formula

$$
k_{N}=\operatorname{lcm}\left(4,2^{r-1} \frac{24}{(24, \sigma(N))}\right)
$$

where lcm denotes the least common multiple and $(\cdot, \cdot)$ stands for the greatest common divisor. 
Proof. From [7], one has that the volume of the surface $X_{N}$ is given by

$$
\operatorname{Vol}\left(X_{N}\right)=\frac{\pi \sigma(N)}{6 \cdot 2^{r-1}}
$$

where $r$ is the number of (distinct) prime factors of $N$. By combining (15) with (11), we have that

$$
k_{N} \cdot \frac{\sigma(N)}{24 \cdot 2^{r-1}}=v_{i \infty}\left(f_{N}\right)
$$

and hence $2^{r-1}(24 /(24, \sigma(N))) \mid k_{N}$.

On the other hand, the cusp form $f_{N}$ does not vanish at order-two elliptic points. As proven above, every surface $X_{N}$ for a square-free $N$ has at least one order-two elliptic point that is a fixed point of the Atkin-Lehner involution $\tau_{N}: z \mapsto-1 /(N z)$. Since

$$
f_{N}\left(\tau_{N}(i / \sqrt{N})\right)=f_{N}(i / \sqrt{N})=(i)^{k_{N}} f_{N}(i / \sqrt{N}),
$$

it follows that $4 \mid k_{N}$. The smallest $k_{N}$ divisible by both 4 and $2^{r-1}(24 /(24, \sigma(N)))$ is given by (14). Therefore, the proof is complete.

The above proposition, together with Theorem 16 from [7], yields the following corollary.

Corollary 5. Let $\ell_{N}=2^{1-r} k_{N}$, where $k_{N}$ is given by (14). Then the function

$$
\Delta_{N}(z):=\left(\prod_{v \mid N} \eta(v z)\right)^{\ell_{N}}
$$

is the smallest-weight cusp form on $X_{N}$ vanishing at the cusp only. Furthermore, the order of vanishing of $\Delta_{N}$ at the cusp is given by

$$
v_{i \infty}\left(\Delta_{N}\right)=\frac{\sigma(N) \ell_{N}}{24}=k_{N} \cdot \frac{\sigma(N)}{24 \cdot 2^{r-1}}
$$

The next proposition determines the smallest-weight $\widetilde{k}_{N}$ for square-free $N$ such that the space $S_{\widetilde{k}_{N}}^{(N)}$ is not empty.

Proposition 6. Let $N=p_{1} \ldots p_{r}$ be a square-free positive integer, where $N>1$. Then the smallest even integer $\widetilde{k}_{N}$ such that there exists a weight- $\widetilde{k}_{N}$ cusp form on a genus-zero surface $X_{N}$ is equal to 8 for $N \in\{2,3\}$ and equal to 4 for all other $N$.

Proof. When $N=2$, it is immediate that $k=8$ is the smallest number such that $k \cdot\left(\operatorname{Vol}\left(X_{2}\right) / 4 \pi\right) \geqslant 1$. Since $\Delta_{2}$ is a weight-eight cusp form, the assertion is proven when $N=2$.

When $N=3, k=6$ is the smallest number such that $k \cdot\left(\operatorname{Vol}\left(X_{3}\right) / 4 \pi\right) \geqslant 1$. However, if there exists a weight-six cusp form on $X_{3}$, this cusp form also vanishes at order-two elliptic point $e_{2}$ of $X_{3}$. Therefore, the right-hand side of the formula (11) is at least $3 / 2$, while the left-hand side of the same formula with $k=6$ is equal to 1 , which yields a contradiction. This shows that eight is the smallest possible weight of cusp form on $X_{3}$. An example of a weight-eight cusp form on $X_{3}$ is $E_{8}^{(3)}-\left(E_{4}^{(3)}\right)^{2}$, so the case when $N=3$ is complete.

When $N \geqslant 5$, we can construct the weight-four cusp form on $X_{N}$, whether or not the genus is zero, as follows. Let

$$
E_{4, N}(z):=\left(E_{2, N}(z)\right)^{2}
$$


From Proposition 3, it is immediate that $E_{4, N}$ is a weight-four holomorphic form on $\overline{\Gamma_{0}(N)^{+}}$. Recall that for a square-free $N$ with $r$ prime factors, we have the formula

$$
\varphi(N)=(-1)^{r} \sum_{v \mid N} v \mu(v) .
$$

The $q$-expansion (5) implies that $E_{2, N}(z)$ is normalized so that its $q$-expansion has a leading coefficient equal to one. Therefore, the difference

$$
\widetilde{\Delta}_{N}(z):=E_{4}^{(N)}(z)-E_{4, N}(z)
$$

is a weight-four cusp form. By computing the $q$-expansion of $E_{4, N}$, we deduce that the term multiplying $q$ in the $q$-expansion of $E_{4, N}(z)$ is $48 / \varphi(N)$, while the term multiplying $q$ in the $q$-expansion of $E_{4}^{(N)}(z)$ is equal to $240 /\left(1+N^{2}\right)$. In other words, for square-free $N \notin\{2,3\}$, we have the expansion

$$
\widetilde{\Delta}_{N}(z)=48\left(\frac{1}{\varphi(N)}-\frac{5}{N^{2}+1}\right) q+\ldots
$$

The leading coefficient is non-zero whenever $N \geqslant 5$ and hence $\widetilde{\Delta}_{N}(z)$ is a weight-four cusp form on $X_{N}$.

\section{Expressing the Hauptmodul in terms of Eisenstein series}

In this section, we discuss the main results of this article.

Theorem 7. For any square-free $N \geqslant 1$ such that the surface $X_{N}$ has genus zero, there exist effectively computable integers $M_{N}$ and $m_{N}$, and explicitly computable polynomials $P_{N}\left(x_{1}, \ldots, x_{m_{N}-1}\right)$ and $Q_{N}\left(x_{1}, \ldots, x_{m_{N}-1}\right)$ in $m_{N}-1$ variables with integer coefficients, such that the Hauptmodul $j_{N}$ can be written as

$$
j_{N}(z)=\frac{P_{N}\left(E_{4}^{(N)}, \ldots, E_{2 m_{N}}^{(N)}\right)}{Q_{N}\left(E_{4}^{(N)}, \ldots, E_{2 m_{N}}^{(N)}\right)}
$$

and the Kronecker limit function can be written as $\Delta_{N}^{M_{N}}=Q_{N}\left(E_{4}^{(N)}, \ldots, E_{2 m_{N}}^{(N)}\right)$.

Proof. The result follows, because for each square-free level $N$, provided that $X_{N}$ has genus zero, the JST3 algorithm terminates in finite time. As stated, the computer code as well as the output is available on the web site [8]. In the space below, let us describe in further detail the output of the computational algorithm. We remind the reader that the JST2 and the JST3 algorithm are described in $\S 2.5$.

After Gauss elimination, one of the $q$-expansions has a pole of order one. This is the Hauptmodul; see $\S 5$ for explicit examples. Keeping track of the linear algebra, we have an exact expression for the Hauptmodul as a linear combination of holomorphic modular functions (10) with rational coefficients. In other words,

$$
j_{N}(z)=\frac{1}{\left(\Delta_{N}(z)\right)^{M_{N}}} \sum_{b} C_{b} \cdot\left(\prod_{\nu}\left(E_{m_{\nu}}^{(N)}(z)\right)^{b_{\nu}}\right),
$$

where the sum is taken over all $b=\left(b_{1}, \ldots\right)$ such that $\sum_{\nu} b_{\nu} m_{\nu}=k_{N} M_{N}$, where $M_{N}$ is given in the right-hand column of Table 1 and $C_{b} \in \mathbb{Q}$. 
There is also a $q$-expansion which is equal to the constant 1 . Again, by keeping track of the linear algebra, we have an exact expression for the constant 1 as

$$
1=\frac{1}{\left(\Delta_{N}(z)\right)^{M_{N}}} \sum_{b} D_{b} \cdot\left(\prod_{\nu}\left(E_{m_{\nu}}^{(N)}(z)\right)^{b_{\nu}}\right),
$$

where the sum is taken over the same set of $b$ as above and $D_{b} \in \mathbb{Q}$.

By the design of the JST3 algorithm, this exact expression can easily be solved for the $M_{N}$ th power of the Kronecker limit function, showing that

$$
j_{N}(z)=\frac{\sum_{b} C_{b} \cdot\left(\prod_{\nu}\left(E_{m_{\nu}}^{(N)}(z)\right)^{b_{\nu}}\right)}{\sum_{b} D_{b} \cdot\left(\prod_{\nu}\left(E_{m_{\nu}}^{(N)}(z)\right)^{b_{\nu}}\right)} .
$$

After multiplication of both numerator and denominator with the least common multiple of the denominators of the numbers $C_{b}$ and $D_{b}$, we deduce the statement of the theorem.

Remark 8. The polynomials $P_{N}$ and $Q_{N}$ appearing in Theorem 7 are weighted homogeneous in the sense that there exists an integer $M_{N}$ such that the coefficient of the term $\left(x_{1}\right)^{\alpha_{1}} \ldots\left(x_{m_{N}-1}\right)^{\alpha_{m_{N}-1}}$ is non-zero only if $4 \alpha_{1}+6 \alpha_{2}+\ldots+2 m_{N} \alpha_{m_{N}-1}=k_{N} M_{N}$, where $k_{N}$ is the weight of the Kronecker limit function $\Delta_{N}$.

Remark 9. Table 1 provides the data regarding the performance of the JST2 and JST3 algorithms. More precisely, the first columns of data in Table 1 list, for each level $N$ provided that $X_{N}$ has genus zero, the weight $k_{N}$ of the Kronecker limit function and the integer $\kappa_{N}$. To recall, it is shown in [7] that if the $q$-expansion of a holomorphic modular form has integer coefficients out to $q^{\kappa_{N}}$, then all further coefficients are also integral. The columns of data in Table 1 under the heading JST2 algorithm list the integer $M$ such that the JST2 algorithm stops, together with the number of $q$-expansions which are used in the Gauss elimination algorithm as well as the order of the largest pole at $i \infty$ amongst the rational functions considered. The columns of data in Table 1 under the heading JST3 algorithm present similar information.

REMARK 10. Table 2 provides a list of the holomorphic Eisenstein series $E_{m_{\nu}}^{(N)}$ which appear in the expression for the Hauptmodul $j_{N}$ cited in Theorem 7 . For all levels, the highest-weight Eisenstein series has weight 26.

REMARK 11. Expressions that are based on the track record of the linear algebra depend on how the base change is made through Gauss elimination. In particular, there may be linearly dependent functions, some of which survive the Gauss elimination while others get annihilated. We sought to express our results in terms of Eisenstein series whose weights are as small as possible. In other words, in the Gauss elimination we prioritized the holomorphic modular functions accordingly.

By expressing the Hauptmodul in terms of holomorphic Eisenstein series of smallest possible weights, we were able to determine a finite list of holomorphic Eisenstein series which generates the rational function field. Let $G$ denote any modular form of weight $k$ and consider the function

$$
F(z)=G(z)\left(E_{6}^{(N)}(z)\right)^{n_{6}}\left(E_{4}^{(N)}(z)\right)^{n_{4}} /\left(\Delta_{N}(z)\right)^{n M_{N}}
$$

with non-negative integers $n_{6}, n_{4}$, and $n$ such that $k+6 n_{6}+4 n_{4}=k_{N} n M_{N}$. There is a rational function $R$ in one variable such that $F(z)=R\left(j_{N}(z)\right)$. Therefore, we conclude that $G$ can be written as a rational function in terms of the holomorphic Eisenstein series that are listed in Table 2. 
TABle 1. Performance of the JST2 and the JST3 algorithms. For all genus-zero groups $\overline{\Gamma_{0}(N)^{+}}$we list the level $N$, the weight $k_{N}$ of the Kronecker limit function, and the value of $\kappa_{N}$ in the proof of integrality [7] (left); the level $N$, the number of iterations $M$, the number of equations, and the largest order of pole for the JST2 algorithm (middle) and similar for the JST3 algorithm (right).

\begin{tabular}{|c|c|c|c|c|c|c|c|c|c|c|}
\hline \multirow[b]{2}{*}{$N$} & \multirow[b]{2}{*}{$k_{N}$} & \multirow[b]{2}{*}{$\kappa_{N}$} & \multicolumn{4}{|c|}{ JST2 algorithm } & \multicolumn{4}{|c|}{ JST3 algorithm } \\
\hline & & & $N$ & $M$ & $\#\{$ eqs $\}$ & Pole & $N$ & $M_{N}$ & $\#\{$ eqs $\}$ & Pole \\
\hline 1 & 12 & 19 & 1 & 1 & 5 & 1 & 1 & 1 & 4 & 1 \\
\hline 2 & 8 & 47 & 2 & 1 & 3 & 1 & 2 & 1 & 2 & 1 \\
\hline 3 & 12 & 48 & 3 & 1 & 5 & 2 & 3 & 1 & 4 & 2 \\
\hline 5 & 4 & 19 & 5 & 1 & 2 & 1 & 5 & 3 & 4 & 3 \\
\hline 6 & 4 & 60 & 6 & 1 & 2 & 1 & 6 & 3 & 4 & 3 \\
\hline 7 & 12 & 19 & 7 & 1 & 5 & 4 & 7 & 2 & 21 & 8 \\
\hline 10 & 8 & 75 & 10 & 2 & 10 & 6 & 10 & 2 & 7 & 6 \\
\hline 11 & 4 & 19 & 11 & 3 & 8 & 6 & 11 & 9 & 88 & 18 \\
\hline 13 & 12 & 19 & 13 & 2 & 26 & 14 & 13 & 3 & 88 & 21 \\
\hline 14 & 4 & 47 & 14 & 3 & 8 & 6 & 14 & 6 & 21 & 12 \\
\hline 15 & 4 & 96 & 15 & 3 & 8 & 6 & 15 & 5 & 12 & 10 \\
\hline 17 & 4 & 19 & 17 & 4 & 15 & 12 & 17 & 9 & 88 & 27 \\
\hline 19 & 12 & 19 & 19 & 3 & 114 & 30 & 19 & 4 & 320 & 40 \\
\hline 21 & 12 & 53 & 21 & 2 & 26 & 16 & 21 & 2 & 21 & 16 \\
\hline 22 & 4 & 47 & 22 & 4 & 15 & 12 & 22 & 6 & 21 & 18 \\
\hline 23 & 4 & 19 & 23 & 5 & 27 & 20 & 23 & 15 & 1039 & 60 \\
\hline 26 & 8 & 47 & 26 & 3 & 31 & 21 & 26 & 4 & 55 & 28 \\
\hline 29 & 4 & 19 & 29 & 6 & 48 & 30 & 29 & 15 & 1039 & 75 \\
\hline 30 & 4 & 127 & 30 & 4 & 15 & 12 & 30 & 6 & 21 & 18 \\
\hline 31 & 12 & 19 & 31 & 4 & 434 & 64 & 31 & 5 & 1039 & 80 \\
\hline 33 & 4 & 48 & 33 & 5 & 27 & 20 & 33 & 8 & 55 & 32 \\
\hline 34 & 8 & 47 & 34 & 3 & 31 & 27 & 34 & 4 & 55 & 36 \\
\hline 35 & 4 & 19 & 35 & 5 & 27 & 20 & 35 & 7 & 34 & 28 \\
\hline 38 & 4 & 47 & 38 & 5 & 27 & 25 & 38 & 10 & 137 & 50 \\
\hline 39 & 12 & 48 & 39 & 3 & 114 & 42 & 39 & 3 & 88 & 42 \\
\hline 41 & 4 & 19 & 41 & 7 & 82 & 49 & 41 & 21 & 8591 & 147 \\
\hline 42 & 4 & 108 & 42 & 5 & 27 & 20 & 42 & 7 & 34 & 28 \\
\hline 46 & 4 & 47 & 46 & 6 & 48 & 36 & 46 & 14 & 708 & 84 \\
\hline 47 & 4 & 19 & 47 & 8 & 137 & 64 & 47 & 27 & 56224 & 216 \\
\hline 51 & 4 & 48 & 51 & 6 & 48 & 36 & 51 & 11 & 210 & 66 \\
\hline 55 & 4 & 19 & 55 & 6 & 48 & 36 & 55 & 8 & 55 & 48 \\
\hline 59 & 4 & 19 & 59 & 9 & 225 & 90 & 59 & 33 & 310962 & 330 \\
\hline 62 & 4 & 47 & 62 & 7 & 82 & 56 & 62 & 18 & 3094 & 144 \\
\hline 66 & 4 & 60 & 66 & 6 & 48 & 36 & 66 & 8 & 55 & 48 \\
\hline 69 & 4 & 48 & 69 & 7 & 82 & 56 & 69 & 14 & 708 & 112 \\
\hline 70 & 4 & 181 & 70 & 6 & 48 & 36 & 70 & 8 & 55 & 48 \\
\hline 71 & 4 & 19 & 71 & 10 & 362 & 120 & 71 & 39 & 1512301 & 468 \\
\hline 78 & 4 & 81 & 78 & 6 & 48 & 42 & 78 & 9 & 88 & 63 \\
\hline 87 & 4 & 48 & 87 & 7 & 82 & 70 & 87 & 17 & 2167 & 170 \\
\hline 94 & 4 & 47 & 94 & 8 & 137 & 96 & 94 & 26 & 41646 & 312 \\
\hline 95 & 4 & 19 & 95 & 7 & 82 & 70 & 95 & 11 & 210 & 110 \\
\hline 105 & 4 & 181 & 105 & 7 & 82 & 56 & 105 & 9 & 88 & 72 \\
\hline 110 & 4 & 89 & 110 & 7 & 82 & 63 & 110 & 9 & 88 & 81 \\
\hline 119 & 4 & 19 & 119 & 8 & 137 & 96 & 119 & 10 & 137 & 120 \\
\hline
\end{tabular}


TABLE 2. Finite sets of Eisenstein series which include the generators of the holomorphic Eisenstein series on groups $\overline{\Gamma_{0}(N)^{+}}$of genus zero. Listed are level $N$ and finite set.

\begin{tabular}{|c|c|}
\hline$N$ & Finite set \\
\hline 1 & $E_{4}^{(1)}, E_{6}^{(1)}$ \\
\hline 2 & $E_{4}^{(2)}, E_{6}^{(2)}, E_{8}^{(2)}$ \\
\hline 3 & $E_{4}^{(3)}, E_{6}^{(3)}, E_{12}^{(3)}$ \\
\hline 5 & $E_{4}^{(5)}, E_{6}^{(5)}, E_{8}^{(5)}, E_{12}^{(5)}$ \\
\hline 6 & $E_{4}^{(6)}, E_{6}^{(6)}, E_{8}^{(6)}, E_{12}^{(6)}$ \\
\hline 7 & $E_{4}^{(7)}, E_{6}^{(7)}, E_{8}^{(7)}, E_{10}^{(7)}, E_{12}^{(7)}$ \\
\hline 10 & $E_{4}^{(10)}, E_{6}^{(10)}, E_{8}^{(10)}, E_{10}^{(10)}, E_{12}^{(10)}, E_{16}^{(10)}$ \\
\hline 11 & $E_{4}^{(11)}, E_{6}^{(11)}, E_{8}^{(11)}, E_{10}^{(11)}, E_{12}^{(11)}$ \\
\hline 13 & $E_{4}^{(13)}, E_{6}^{(13)}, E_{8}^{(13)}, E_{10}^{(13)}, E_{12}^{(13)}$ \\
\hline 14 & $E_{4}^{(14)}, E_{6}^{(14)}, E_{8}^{(14)}, E_{10}^{(14)}, E_{12}^{(14)}$ \\
\hline 15 & $E_{4}^{(15)}, E_{6}^{(15)}, E_{8}^{(15)}, E_{10}^{(15)}, E_{12}^{(15)}, E_{14}^{(15)}, E_{16}^{(15)}$ \\
\hline 17 & $E_{4}^{(17)}, E_{6}^{(17)}, E_{8}^{(17)}, E_{10}^{(17)}, E_{12}^{(17)}$ \\
\hline 19 & $E_{4}^{(19)}, E_{6}^{(19)}, E_{8}^{(19)}, E_{10}^{(19)}, E_{12}^{(19)}$ \\
\hline 21 & $E_{4}^{(21)}, E_{6}^{(21)}, E_{8}^{(21)}, E_{10}^{(21)}, E_{12}^{(21)}, E_{14}^{(21)}, E_{16}^{(21)}$ \\
\hline 22 & $E_{4}^{(22)}, E_{6}^{(22)}, E_{8}^{(22)}, E_{10}^{(22)}, E_{12}^{(22)}, E_{14}^{(22)}, E_{16}^{(22)}, E_{18}^{(22)}$ \\
\hline 23 & $E_{4}^{(23)}, E_{6}^{(23)}, E_{8}^{(23)}, E_{10}^{(23)}, E_{12}^{(23)}$ \\
\hline 26 & $E_{4}^{(26)}, E_{6}^{(26)}, E_{8}^{(26)}, E_{10}^{(26)}, E_{12}^{(26)}, E_{14}^{(26)}$ \\
\hline 29 & $E_{4}^{(29)}, E_{6}^{(29)}, E_{8}^{(29)}, E_{10}^{(29)}, E_{12}^{(29)}$ \\
\hline 30 & $E_{4}^{(30)}, E_{6}^{(30)}, E_{8}^{(30)}, E_{10}^{(30)}, E_{12}^{(30)}, E_{14}^{(30)}, E_{16}^{(30)}, E_{18}^{(30)}$ \\
\hline 31 & $E_{4}^{(31)}, E_{6}^{(31)}, E_{8}^{(31)}, E_{10}^{(31)}, E_{12}^{(31)}$ \\
\hline 33 & $E_{4}^{(33)}, E_{6}^{(33)}, E_{8}^{(33)}, E_{10}^{(33)}, E_{12}^{(33)}, E_{14}^{(33)}$ \\
\hline 34 & $E_{4}^{(34)}, E_{6}^{(34)}, E_{8}^{(34)}, E_{10}^{(34)}, E_{12}^{(34)}, E_{14}^{(34)}, E_{16}^{(34)}$ \\
\hline 35 & $E_{4}^{(35)}, E_{6}^{(35)}, E_{8}^{(35)}, E_{10}^{(35)}, E_{12}^{(35)}, E_{14}^{(35)}, E_{16}^{(35)}, E_{18}^{(35)}$ \\
\hline 38 & $E_{4}^{(38)}, E_{6}^{(38)}, E_{8}^{(38)}, E_{10}^{(38)}, E_{12}^{(38)}, E_{14}^{(38)}$ \\
\hline 39 & $E_{4}^{(39)}, E_{6}^{(39)}, E_{8}^{(39)}, E_{10}^{(39)}, E_{12}^{(39)}, E_{14}^{(39)}$ \\
\hline 41 & $E_{4}^{(41)}, E_{6}^{(41)}, E_{8}^{(41)}, E_{10}^{(41)}, E_{12}^{(41)}$ \\
\hline & $E_{4}^{(42)}, E_{6}^{(42)}, E_{8}^{(42)}, E_{10}^{(42)}, E_{12}^{(42)}, E_{14}^{(42)}, E_{16}^{(42)}, E_{18}^{(42)}$ \\
\hline 46 & $E_{4}^{(46)}, E_{6}^{(46)}, E_{8}^{(46)}, E_{10}^{(46)}, E_{12}^{(46)}$ \\
\hline 47 & $E_{4}^{(47)}, E_{6}^{(47)}, E_{8}^{(47)}, E_{10}^{(47)}, E_{12}^{(47)}$ \\
\hline 51 & $E_{4}^{(51)}, E_{6}^{(51)}, E_{8}^{(51)}, E_{10}^{(51)}, E_{12}^{(51)}, E_{14}^{(51)}$ \\
\hline 55 & $E_{4}^{(55)}, E_{6}^{(55)}, E_{8}^{(55)}, E_{10}^{(55)}, E_{12}^{(55)}, E_{14}^{(55)}, E_{16}^{(55)}, E_{18}^{(55)}, E_{20}^{(55)}, E_{22}^{(55)}$ \\
\hline 59 & $E_{4}^{(59)}, E_{6}^{(59)}, E_{8}^{(59)}, E_{10}^{(59)}, E_{12}^{(59)}$ \\
\hline 62 & $E_{4}^{(62)}, E_{6}^{(62)}, E_{8}^{(62)}, E_{10}^{(62)}, E_{12}^{(62)}$ \\
\hline 66 & $E_{4}^{(66)}, E_{6}^{(66)}, E_{8}^{(66)}, E_{10}^{(66)}, E_{12}^{(66)}, E_{14}^{(66)}, E_{16}^{(66)}, E_{18}^{(66)}, E_{20}^{(66)}, E_{22}^{(66)}$ \\
\hline 69 & $E_{4}^{(69)}, E_{6}^{(69)}, E_{8}^{(69)}, E_{10}^{(69)}, E_{12}^{(69)}$ \\
\hline 70 & $E_{4}^{(70)}, E_{6}^{(70)}, E_{8}^{(70)}, E_{10}^{(70)}, E_{12}^{(70)}, E_{14}^{(70)}, E_{16}^{(70)}, E_{18}^{(70)}, E_{20}^{(70)}, E_{22}^{(70)}$ \\
\hline 71 & $E_{4}^{(71)}, E_{6}^{(71)}, E_{8}^{(71)}, E_{10}^{(71)}, E_{12}^{(71)}$ \\
\hline 78 & $E_{4}^{(78)}, E_{6}^{(78)}, E_{8}^{(78)}, E_{10}^{(78)}, E_{12}^{(78)}, E_{14}^{(78)}, E_{16}^{(78)}, E_{18}^{(78)}$ \\
\hline 87 & $E_{4}^{(87)}, E_{6}^{(87)}, E_{8}^{(87)}, E_{10}^{(87)}, E_{12}^{(87)}$ \\
\hline 94 & $E_{4}^{(94)}, E_{6}^{(94)}, E_{8}^{(94)}, E_{10}^{(94)}, E_{12}^{(94)}$ \\
\hline 95 & $E_{4}^{(95)}, E_{6}^{(95)}, E_{8}^{(95)}, E_{10}^{(95)}, E_{12}^{(95)}, E_{14}^{(95)}, E_{16}^{(95)}$ \\
\hline 105 & $E_{4}^{(105)}, E_{6}^{(105)}, E_{8}^{(105)}, E_{10}^{(105)}, E_{12}^{(105)}, E_{14}^{(105)}, E_{16}^{(105)}, E_{18}^{(105)}, E_{20}^{(105)}$ \\
\hline 110 & $E_{4}^{(110)}, E_{6}^{(110)}, E_{8}^{(110)}, E_{10}^{(110)}, E_{12}^{(110)}, E_{14}^{(110)}, E_{16}^{(110)}, E_{18}^{(110)}, E_{20}^{(110)}, E_{22}^{(110)}, E_{24}^{(110)}, E_{26}^{(110)}$ \\
\hline & $E_{4}^{(119)}, E_{6}^{(119)}, E_{8}^{(119)}, E_{10}^{(119)}, E_{12}^{(119)}, E_{14}^{(119)}, E_{16}^{(119)}, E_{18}^{(119)}, E_{20}^{(119)}, E_{22}^{(119)}, E_{24}^{(119)}$ \\
\hline
\end{tabular}


REMARK 12 . We note that the sets in Table 2 are not necessarily minimal sets of generators. A specific example in the case $N=2$ is discussed below. As stated in the introduction, our goal was to determine a set of generators of the function field. Indeed, it seems to be a difficult problem to determine the structure of the ring of modular forms in any setting when $M_{N}>1$, meaning when there is an expression for the $M_{N}$ th power of the Kronecker limit function in terms of holomorphic Eisenstein series yet no apparent expression for any smaller power of the Kronecker limit function.

\section{Examples}

In this section, we will present a number of specific formulae for various levels. It seems as if each level has its own idiosyncratic characteristics, so we choose various examples, which, in our opinion, depict some of the most comprehensible and quantifiable nuances.

\section{1. $\quad N=2$}

We will cite specific results here, referring the reader to the article [9] for additional information and proofs. The Kronecker limit function can be written as

$$
\Delta_{2}(z)=\frac{17}{1152}\left(E_{4}^{(2)}(z)\right)^{2}-\frac{17}{1152} E_{8}^{(2)}(z)
$$

In addition, one has that

$$
j_{2}(z) \Delta_{2}(z)=-\frac{77}{144}\left(E_{4}^{(2)}(z)\right)^{2}+\frac{221}{144} E_{8}^{(2)}(z)
$$

By arguing as in $[\mathbf{1 0}]$, one can prove a dimension formula for the space of automorphic forms of weight $2 k$, namely that

$$
\operatorname{dim} \mathcal{T}_{2 k}^{(2)}= \begin{cases}\left\lfloor\frac{k}{4}\right\rfloor & \text { if } k \text { is congruent to } 1 \text { modulo } 4, k \geqslant 0, \\ \left\lfloor\frac{k}{4}\right\rfloor+1 & \text { if } k \text { is not congruent to } 1 \text { modulo } 4, k \geqslant 0\end{cases}
$$

The space $\mathcal{T}_{2 k}^{(2)}$ is generated by the set of monomials $\left(E_{4}^{(2)}(z)\right)^{l}\left(E_{6}^{(2)}(z)\right)^{m}\left(E_{8}^{(2)}(z)\right)^{n}$, where $l, m, n$ are non-negative integers such that $4 l+6 m+8 n=2 k$. The dimension formula (17) yields some interesting number-theoretical formulae. For example, since $\operatorname{dim} \mathcal{T}_{10}^{(2)}=1$, we see that $E_{10}^{(2)}(z)=E_{6}^{(2)}(z) E_{4}^{(2)}(z)$. By equating the $q$-expansions (8) for $k \in\{2,3,5\}$, one obtains the following summation formula for the generalized sum of divisors:

$$
A_{9}^{(2)}(n)=336 \sum_{j=1}^{n-1} A_{3}^{(2)}(j) A_{5}^{(2)}(n-j)+7 A_{5}^{(2)}(n)-6 A_{3}^{(2)}(n),
$$

where $A_{2 k-1}^{(2)}(n)=\sigma_{2 k-1}(n)+2^{k} \delta(n) \sigma_{2 k-1}(n / 2)$ for $k=1,2, \ldots$ and $\delta(n)=1$ for even positive integers $n$ and $\delta(n)=0$, otherwise.

Analogously, using formula (16), the $q$-expansion (8), and the $q$-expansion for the delta function, $\Delta(z)=\sum_{n=1}^{\infty} \tau(n) q^{n}$, where $\tau(n)$ is the Ramanujan function, one obtains relations involving $\tau, \sigma_{3}$, and $\sigma_{7}$. 


\section{2. $\quad N=3$}

As with the case $N=2$, we refer the reader to [9] for additional information and proofs. The Kronecker limit function vanishes to order two at $i \infty$ and has weight twelve. The smallestweight cusp form has weight eight, but it vanishes to order one at $i \infty$, and, consequently, it vanishes elsewhere. The Kronecker limit function can be written as

$$
\Delta_{3}(z)=-\frac{25}{3456}\left(E_{4}^{(3)}(z)\right)^{3}-\frac{1049}{72900}\left(E_{6}^{(3)}(z)\right)^{2}+\frac{50443}{2332800} E_{12}^{(3)}(z) .
$$

and the Hauptmodul is given by

$$
j_{3}(z) \Delta_{3}(z)=\frac{541}{1728}\left(E_{4}^{(3)}(z)\right)^{3}+\frac{14461}{24300}\left(E_{6}^{(3)}(z)\right)^{2}-\frac{353101}{388800} E_{12}^{(3)}(z)
$$

The dimension formula for the space of automorphic forms of weight $2 k$ is

$$
\operatorname{dim} \mathcal{T}_{2 k}^{(3)}= \begin{cases}\left\lfloor\frac{k}{3}\right\rfloor & \text { if } k \text { is congruent to } 1 \text { or } 3 \text { modulo } 6, k \geqslant 0, \\ \left\lfloor\frac{k}{3}\right\rfloor+1 & \text { if } k \text { is not congruent to } 1 \text { or } 3 \text { modulo } 6, k \geqslant 0\end{cases}
$$

We note that the forms $E_{8}^{(3)}(z)-\left(E_{4}^{(3)}(z)\right)^{2}$ and $E_{10}^{(3)}(z)-E_{4}^{(3)}(z) E_{6}^{(3)}(z)$ are cusp forms which vanish at elliptic points on $X_{3}$; see Appendix B of [9]. In other words, there are cusp forms of weight smaller than the weight of the Kronecker limit function, but these forms necessarily vanish at some point in the interior of $X_{3}$, whereas the Kronecker limit function vanishes at $i \infty$ only.

Finally, let us explain why $E_{8}^{(3)}$ does not appear in Table 2. The information in Appendix B of [9] describes the zeros of small-weight holomorphic forms. In particular, we conclude from the information provided that

$$
\frac{E_{8}^{(3)}(z)}{E_{8}^{(3)}(z)-\left(E_{4}^{(3)}(z)\right)^{2}}=c_{1} j_{3}(z)+c_{2}
$$

for some explicitly computable constants $c_{1}$ and $c_{2}$. From this, we get that

$$
E_{8}^{(3)}(z)=\left(E_{4}^{(3)}(z)\right)^{2} \frac{c_{1} j_{3}(z)+c_{2}}{c_{1} j_{3}(z)+c_{2}-1} .
$$

When combining (19), (18), and (20), we get a formula which expresses $E_{8}^{(3)}$ as a rational function involving $E_{4}^{(3)}, E_{6}^{(3)}$, and $E_{12}^{(3)}$, as asserted by Table 2 .

\section{3. $\quad N=5$}

In the case $N=5$, the surface $X_{5}$ has genus zero, three order-two elliptic elements $e_{1}=i / \sqrt{5}$, $e_{2}=2 / 5+i / 5, e_{3}=1 / 2+i /(2 \sqrt{5})$, and one cusp; hence, $\operatorname{Vol}_{\text {hyp }}\left(X_{5}\right)=\pi$. Its Kronecker limit function has weight four, which is minimal, and the function vanishes at $i \infty$ to order one, which is also minimal. As a result, we have that the mapping $f \mapsto \Delta_{5} f$ is an isometry between the spaces $\mathcal{T}_{2 k-4}^{(5)}$ and $S_{2 k}^{(5)}$; therefore, we arrive at the dimension formula

$$
\operatorname{dim} \mathcal{T}_{2 k}^{(2)}= \begin{cases}\left\lfloor\frac{k}{2}\right\rfloor & \text { if } k \text { is congruent to } 1 \text { modulo } 2, k \geqslant 0, \\ \left\lfloor\frac{k}{2}\right\rfloor+1 & \text { if } k \text { is not congruent to } 0 \text { modulo } 2, k \geqslant 0 .\end{cases}
$$


The space $\mathcal{T}_{2 k}^{(5)}$ is generated by the set of monomials $\left(E_{4}^{(5)}(z)\right)^{l}\left(\Delta_{5}(z)\right)^{m}\left(E_{6}^{(5)}(z)\right)^{n}$, where $l, m, n$ are non-negative integers such that $4 l+4 m+6 n=2 k$. From the output of the JST2 algorithm, we have that

$$
j_{5}(z) \Delta_{5}(z)=E_{4}^{(5)}(z)-\frac{172}{13} \Delta_{5}(z) .
$$

The analysis of $\Delta_{5}^{3}$ differs between the JST2 and the JST3 algorithms. From JST2, we have that $\Delta_{5}^{3}$ is a rational function in the holomorphic Eisenstein series of weights four, six, eight, and twelve. From JST3, we have that $\Delta_{5}^{3}$ is a polynomial in the holomorphic Eisenstein series of weights four, six, eight, and twelve. Namely, from the output of the JST3 algorithm, we have that

$$
\begin{aligned}
j_{5}(z)\left(\Delta_{5}(z)\right)^{3}= & \frac{10330419229}{11016000000}\left(E_{4}^{(5)}(z)\right)^{3}+\frac{36659}{2448000}\left(E_{6}^{(5)}(z)\right)^{2} \\
& -\frac{28493266087}{11016000000} E_{8}^{(5)}(z) E_{4}^{(5)}(z)+\frac{2999646893}{1836000000} E_{12}^{(5)}(z)
\end{aligned}
$$

and

$$
\begin{aligned}
\left(\Delta_{5}(z)\right)^{3}= & -\frac{9383387}{162000000}\left(E_{4}^{(5)}(z)\right)^{3}-\frac{13}{9000}\left(E_{6}^{(5)}(z)\right)^{2} \\
& +\frac{3226717}{20250000} E_{8}^{(5)}(z) E_{4}^{(5)}(z)-\frac{5398783}{54000000} E_{12}^{(5)}(z) .
\end{aligned}
$$

\section{4. $\quad N=6$}

Topologically, $X_{5}$ and $X_{6}$ are identical, with the same number of cusps, elliptic points of order two, and, consequently, the same hyperbolic volume. The JST2 and JST3 algorithms performed similarly in both cases, as one can see from Tables 1 and 2. All comments above regarding the holomorphic function theory for $X_{5}$ hold for $X_{6}$. However, as shown in [6], the analytic function theories of $X_{5}$ and $X_{6}$ are different. Specifically, the counting functions for the analytic Maass forms, when ordered by their Laplacian eigenvalues, are shown to be equal in their leading term but unequal in lower order terms.

\section{5. $\quad N=17$}

As we stated in the introduction, as $N$ becomes larger, the formulae become massive. In our last example for $N=17$, the Kronecker limit function has weight four and vanishes at $i \infty$ to order four. From the JST3 algorithm, we have the following formulae:

$$
\begin{aligned}
j_{17}(z) & \left(\Delta_{17}(z)\right)^{9}=\frac{81682801889356820001790224970058471917613108127362192461613220533}{3269846855773492420944242299705431901325975126578932604974661632}\left(E_{4}^{(17)}(z)\right)^{9} \\
& -\frac{57998022455299820152689336251300228068357304045275286805301}{1197457521190948626327504142387996791894290229520024731648}\left(E_{6}^{(17)}(z)\right)^{2}\left(E_{4}^{(17)}(z)\right)^{6} \\
& +\frac{40497436515338798408532045523225489025965457561330556316291}{1852774239194857326466652706713276353684752025138495488000}\left(E_{6}^{(17)}(z)\right)^{4}\left(E_{4}^{(17)}(z)\right)^{3} \\
& -\frac{3758480257690225061233693208729793594924453574315163}{550341367907988517797569501748755788899740024832000}\left(E_{6}^{(17)}(z)\right)^{6} \\
& -\frac{19414695740146736017085565287911573267947533788487530546931336997391}{235020242758719767755367415291327917907804462222860780982553804800} E_{8}^{(17)}(z)\left(E_{4}^{(17)}(z)\right)^{7} \\
& +\frac{87392429573930662513617849766871793131053840056808626436739811257}{429213680251880648249264766037197600094609654143583864750080000} E_{8}^{(17)}(z)\left(E_{6}^{(17)}(z)\right)^{2}\left(E_{4}^{(17)}(z)\right)^{4} \\
& -\frac{5203291809002722923420727059042670529678338299681100572348497}{159222786180808051493227966983172186644783377160339456000000} E_{8}^{(17)}(z)\left(E_{6}^{(17)}(z)\right)^{4} E_{4}^{(17)}(z) \\
& -\frac{34080218817076206028500441413178574451045377552516243513916285865231}{546558704090045971524110268119367250948382470285722746471055360000}\left(E_{8}^{(17)}(z)\right)^{2}\left(E_{4}^{(17)}(z)\right)^{5} \\
& -\frac{16613503534705813629198888518084937494696284987808069450102921171}{95380817833751255166503281341599466687691034254129747722240000}\left(E_{8}^{(17)}(z)\right)^{2}\left(E_{6}^{(17)}(z)\right)^{2}\left(E_{4}^{(17)}(z)\right)^{2} \\
& +\frac{2084310764069464266375452181379302123943671896630614730410490282708941}{24481275287366642474517439092846658115396298148214664685682688000000}\left(E_{8}^{(17)}(z)\right)^{3}\left(E_{4}^{(17)}(z)\right)^{3} \\
& -\frac{9922136522478992021059089148544040599546174236808342061149389}{600269903901646354129469435526559143650833331894479749120000}\left(E_{8}^{(17)}(z)\right)^{3}\left(E_{6}^{(17)}(z)\right)^{2} \\
& +\frac{39971724261482388723963548784518805970985444209456555554807081177551}{580296895700542636433005963682291155327912252402125385142108160000}\left(E_{8}^{(17)}(z)\right)^{4} E_{4}^{(17)}(z) \\
& -\frac{12179813594881425731721530954876395006064827564865712237231709007}{111595556865488968544808839169671376024598510077331804835020800} E_{10}^{(17)}(z) E_{6}^{(17)}(z)\left(E_{4}^{(17)}(z)\right)^{5}
\end{aligned}
$$


$-\frac{1625258630148098158844608861059428762679867410760523654188493}{305707749467151458866997696607690598357984084147851755520000} E_{10}^{(17)}(z)\left(E_{6}^{(17)}(z)\right)^{3}\left(E_{4}^{(17)}(z)\right)^{2}$

$+\frac{201956165169824936446796453214912198922237546648448042931247350643}{697472230409306053405055244810446100153740687983323780218880000} E_{10}^{(17)}(z) E_{8}^{(17)}(z) E_{6}^{(17)}(z)\left(E_{4}^{(17)}(z)\right)^{3}$

$+\frac{2813492092804509777019550484166164259166371672470004179865987}{110819059181842403839286665020287841904769230503596261376000} E_{10}^{(17)}(z) E_{8}^{(17)}(z)\left(E_{6}^{(17)}(z)\right)^{3}$

$-\frac{298150566267220507427939657298379519874877536521040468383984890437}{2789888921637224213620220979241784400614962751933295120875520000} E_{10}^{(17)}(z)\left(E_{8}^{(17)}(z)\right)^{2} E_{6}^{(17)}(z) E_{4}^{(17)}(z)$

$+\frac{270142921637107712433606444209937936587102913556256973385136064101}{1339146682385867622537706070036056512295182120927981658020249600}\left(E_{10}^{(17)}(z)\right)^{2}\left(E_{4}^{(17)}(z)\right)^{4}$

$-\frac{118581244701243654625492701946386671875131600350921745366238181}{1324733581024322988423656685299992592884597697974024273920000}\left(E_{10}^{(17)}(z)\right)^{2}\left(E_{6}^{(17)}(z)\right)^{2} E_{4}^{(17)}(z)$

$-\frac{622123658423176240663440801764445330920253599234807877389715511211}{1287641040755641944747794298111592800283828962430751594250240000}\left(E_{10}^{(17)}(z)\right)^{2} E_{8}^{(17)}(z)\left(E_{4}^{(17)}(z)\right)^{2}$

$-\frac{41865928593946018666000515901728601665582738769261235242020892533923}{970881344729754026339836900776140971414007037672786702064680960000}\left(E_{10}^{(17)}(z)\right)^{2}\left(E_{8}^{(17)}(z)\right)^{2}$

$+\frac{226293023118065631604956427915797705760362816981994085929537}{2676229456614793916007387243030288066433530702977826816000}\left(E_{10}^{(17)}(z)\right)^{3} E_{6}^{(17)}(z)$

$+\frac{65939988441096018663885334259995469677}{1934740380512325216435446698160947200} E_{12}^{(17)}(z)\left(E_{4}^{(17)}(z)\right)^{6}$

$-\frac{47508694350054116027131578232203571279280588142292406574968917}{496775092884121120658871256987497222331724136740259102720000} E_{12}^{(17)}(z)\left(E_{6}^{(17)}(z)\right)^{2}\left(E_{4}^{(17)}(z)\right)^{3}$

$+\frac{83239130800439493554048989758304142656207424379566457431}{2818127349459872237687554172747939125917634265088000000} E_{12}^{(17)}(z)\left(E_{6}^{(17)}(z)\right)^{4}$

$+\frac{18888098569562683617871650219704377045625972851708713833077441}{99355018576824224131774251397499444466344827348051820544000} E_{12}^{(17)}(z) E_{10}^{(17)}(z) E_{6}^{(17)}(z)\left(E_{4}^{(17)}(z)\right)^{2}$

$-\frac{6458100747185096513157918629463271052359}{48368509512808130410886167454023680000}\left(E_{12}^{(17)}(z)\right)^{2}\left(E_{4}^{(17)}(z)\right)^{3}$

$+\frac{13456181814083822984196529705199819074619}{77183791775757654910988565086208000000}\left(E_{12}^{(17)}(z)\right)^{3}$

and

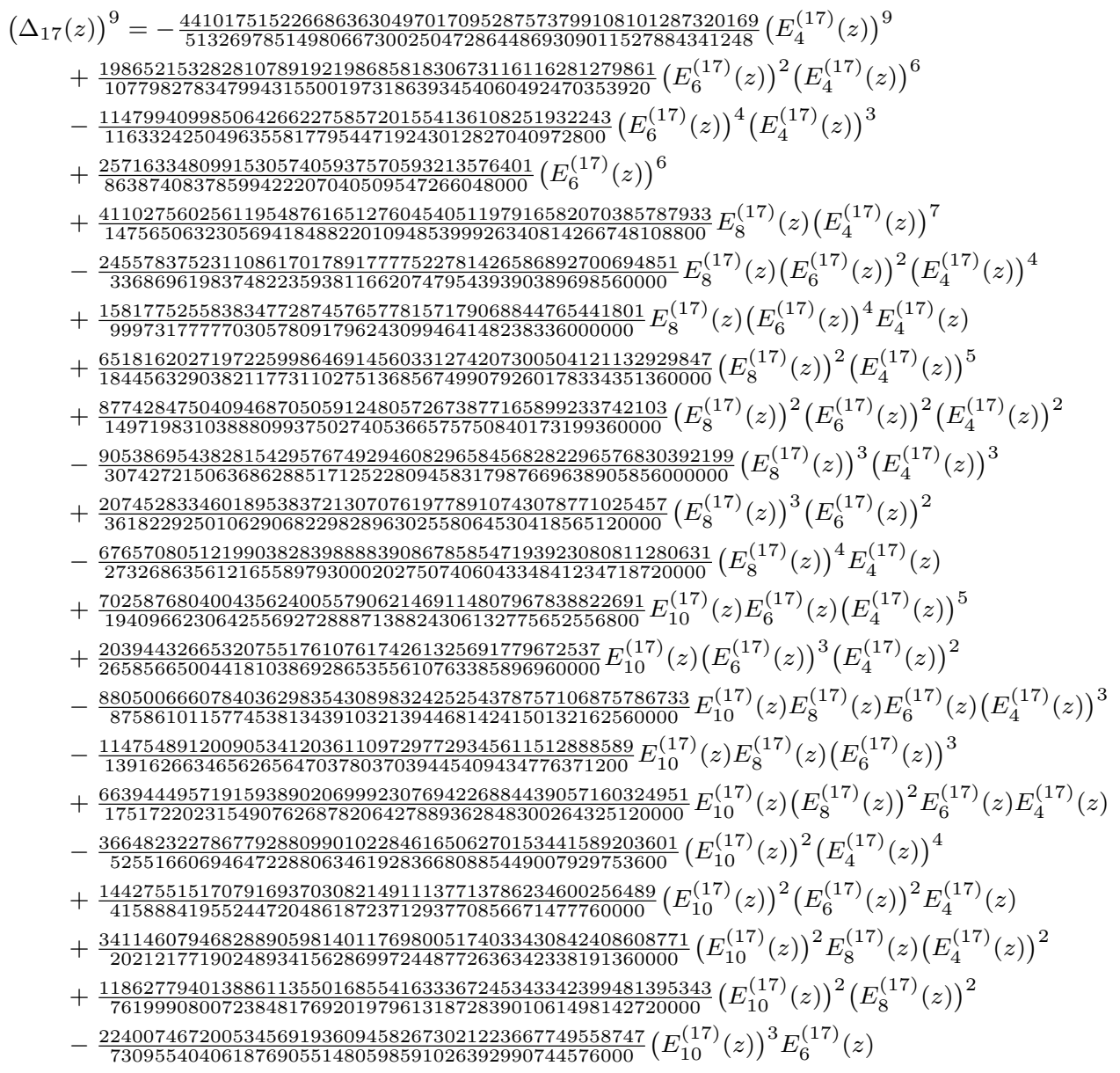




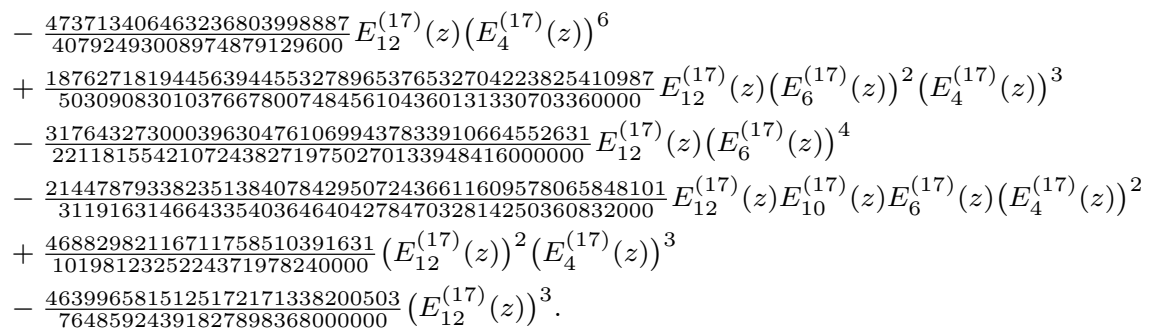

Using the exact identity of the Hauptmodul in terms of Eisenstein series, we can read off that $E_{4}^{(17)}(z), E_{6}^{(17)}(z), E_{8}^{(17)}(z), E_{10}^{(17)}(z)$, and $E_{12}^{(17)}(z)$ generate the holomorphic Eisenstein series $E_{k}^{(17)}(z)$ for all even $k \geqslant 4$.

\section{Concluding remarks}

\subsection{Known relations for Hauptmoduli}

In [2], the authors computed expressions for $j_{N}$, up to an additive constant. The authors call their function $t_{N}$. The data from [2] relates to genus-zero groups $\Gamma_{0}(N)^{+}$with square-free level $N$ as given in Table 3, using the Dedekind eta function together with $\theta(a, b, c)$, which is the theta function defined by the series

$$
\theta(a, b, c)=\sum_{(x, y) \in \mathbb{Z}^{2}} q^{\left(a x^{2}+b x y+c y^{2}\right) / 2} .
$$

Additionally, one has, in the notation of [2], the functions $\theta_{x}(a, b, c)$ and $\theta_{y}(a, b, c)$, which are defined by the same series which defines $\theta(a, b, c)$ except that one restricts the sum to odd values of $x$ and $y$, respectively. By combining our results with the relations for the Hauptmoduli in Table 3, it is possible to deduce many potentially interesting relations between classical Eisenstein series $E_{k}(z)$, eta functions, and theta functions.

For example, let us take $N=17$. In the notation of Theorem 7 , one has $M_{17}=9$ and the Hauptmodul $j_{17}(z)$ is given as a rational function of the form

$$
j_{17}(z)=\frac{P_{17}\left(E_{4}^{(17)}, E_{6}^{(17)}, E_{8}^{(17)}, E_{10}^{(17)}, E_{12}^{(17)}\right)}{Q_{17}\left(E_{4}^{(17)}, E_{6}^{(17)}, E_{8}^{(17)}, E_{10}^{(17)}, E_{12}^{(17)}\right)},
$$

where $P_{17}$ and $Q_{17}$ denote polynomials of degree nine in five variables with integer coefficients, where coefficients are non-zero only if the sum of products of weights and corresponding degrees is equal to 36 .

In a sense, this result is a direct analogue of formula (2) expressing the classical $j$-invariant for $\operatorname{PSL}(2, \mathbb{Z})$ in terms of classical holomorphic Eisenstein series.

Furthermore, formula (7) implies that the Eisenstein series $E_{2 k}^{(17)}$ for $k=2,3,4,5,6$ may be expressed as a linear combination of dilations of the series $E_{2 k}$; hence, the function

$$
\left(\frac{\theta_{x}\left(\frac{1}{2}, 0, \frac{17}{2}\right)-\theta_{y}\left(\frac{1}{2}, 0, \frac{17}{2}\right)}{2 \eta(z) \eta(17 z)}\right)^{2}
$$

is a rational function in the Eisenstein series $E_{4}(z), E_{4}(17 z), E_{6}(z), E_{6}(17 z), E_{8}(z), E_{8}(17 z)$, $E_{10}(z), E_{10}(17 z), E_{12}(z)$, and $E_{12}(17 z)$ with integer coefficients.

Proceeding in a similar manner, for example when $N=29$ or $N=47$, we obtain other relations between theta functions, eta functions, and holomorphic Eisenstein series $E_{2 k}$. 
TABLE 3. Known expressions of the Hauptmoduli $j_{N}$ for the genus-zero groups $\Gamma_{0}(N)^{+}$.

\begin{tabular}{|c|c|}
\hline$N$ & Formula for $t_{N}=j_{N}+$ const. \\
\hline 2 & $t_{2}=\left(\frac{\eta(z)}{\eta(2 z)}\right)^{24}+4096\left(\frac{\eta(2 z)}{\eta(z)}\right)^{24}$ \\
\hline 3 & $t_{3}=\left(\frac{\eta(z)}{\eta(3 z)}\right)^{12}+729\left(\frac{\eta(3 z)}{\eta(z)}\right)^{12}$ \\
\hline 5 & $t_{5}=\left(\frac{\eta(z)}{\eta(5 z)}\right)^{6}+125\left(\frac{\eta(5 z)}{\eta(z)}\right)^{6}$ \\
\hline \multirow[t]{2}{*}{6} & $t_{6}=\left(\frac{\eta(z) \eta(2 z)}{\eta(3 z) \eta(6 z)}\right)^{4}+81\left(\frac{\eta(3 z) \eta(6 z)}{\eta(z) \eta(2 z)}\right)^{4}=\left(\frac{\eta(z) \eta(3 z)}{\eta(2 z) \eta(6 z)}\right)^{6}+64\left(\frac{\eta(2 z) \eta(6 z)}{\eta(z) \eta(3 z)}\right)^{6}+c_{1}$ \\
\hline & $=\left(\frac{\eta(2 z) \eta(3 z)}{\eta(z) \eta(6 z)}\right)^{12}+\left(\frac{\eta(z) \eta(6 z)}{\eta(2 z) \eta(3 z)}\right)^{12}+c_{2}$ \\
\hline 7 & $t_{7}=\left(\frac{\eta(z)}{\eta(7 z)}\right)^{4}+49\left(\frac{\eta(7 z)}{\eta(z)}\right)^{4}$ \\
\hline \multirow[t]{2}{*}{10} & $t_{10}=\left(\frac{\eta(z) \eta(2 z)}{\eta(5 z) \eta(10 z)}\right)^{2}+25\left(\frac{\eta(5 z) \eta(10 z)}{\eta(z) \eta(2 z)}\right)^{2}=\left(\frac{\eta(z) \eta(5 z)}{\eta(2 z) \eta(10 z)}\right)^{4}+16\left(\frac{\eta(2 z) \eta(10 z)}{\eta(z) \eta(5 z)}\right)^{4}+c_{1}$ \\
\hline & $=\left(\frac{\eta(2 z) \eta(5 z)}{\eta(z) \eta(10 z)}\right)^{6}+\left(\frac{\eta(z) \eta(10 z)}{\eta(2 z) \eta(5 z)}\right)^{6}+c_{2}$ \\
\hline 11 & $t_{11}=\left(\frac{\theta(2,2,6)}{\eta(z) \eta(11 z)}\right)^{2}=\left(\frac{\eta(z) \eta(11 z)}{\eta(2 z) \eta(22 z)}\right)^{2}+16\left(\frac{\eta(2 z) \eta(22 z)}{\eta(z) \eta(11 z)}\right)^{2}+16\left(\frac{\eta(2 z) \eta(22 z)}{\eta(z) \eta(11 z)}\right)^{4}+c_{1}$ \\
\hline 13 & $t_{13}=\left(\frac{\eta(z)}{\eta(13 z)}\right)^{2}+13\left(\frac{\eta(13 z)}{\eta(z)}\right)^{2}$ \\
\hline 14 & $t_{14}=\left(\frac{\eta(z) \eta(7 z)}{\eta(2 z) \eta(14 z)}\right)^{3}+8\left(\frac{\eta(2 z) \eta(14 z)}{\eta(z) \eta(7 z)}\right)^{3}=\left(\frac{\eta(2 z) \eta(7 z)}{\eta(z) \eta(14 z)}\right)^{4}+\left(\frac{\eta(z) \eta(14 z)}{\eta(2 z) \eta(7 z)}\right)^{4}+c_{1}$ \\
\hline 15 & $t_{15}=\left(\frac{\eta(z) \eta(5 z)}{\eta(3 z) \eta(15 z)}\right)^{2}+9\left(\frac{\eta(3 z) \eta(15 z)}{\eta(z) \eta(5 z)}\right)^{2}=\left(\frac{\eta(3 z) \eta(5 z)}{\eta(z) \eta(15 z)}\right)^{3}+\left(\frac{\eta(z) \eta(15 z)}{\eta(3 z) \eta(5 z)}\right)^{3}+c_{1}$ \\
\hline 17 & $t_{17}=\left(\frac{\theta_{x}\left(\frac{1}{2}, 0, \frac{17}{2}\right)-\theta_{y}\left(\frac{1}{2}, 0, \frac{17}{2}\right)}{2 \eta(z) \eta(17 z)}\right)^{2}$ \\
\hline 19 & $t_{19}=\left(\frac{2 \theta(2,2,10)}{\theta(1,2,20)-\theta(4,2,5)}\right)^{2}$ \\
\hline 21 & $t_{21}=\left(\frac{\eta(z) \eta(3 z)}{\eta(7 z) \eta(21 z)}\right)+7\left(\frac{\eta(7 z) \eta(21 z)}{\eta(z) \eta(3 z)}\right)=\left(\frac{\eta(3 z) \eta(7 z)}{\eta(z) \eta(21 z)}\right)^{2}+\left(\frac{\eta(z) \eta(21 z)}{\eta(3 z) \eta(7 z)}\right)^{2}+c_{1}$ \\
\hline 22 & $t_{22}=\left(\frac{\eta(z) \eta(11 z)}{\eta(2 z) \eta(22 z)}\right)^{2}+4\left(\frac{\eta(2 z) \eta(22)}{\eta(z) \eta(11 z)}\right)^{2}$ \\
\hline 23 & $t_{23}=\left(\frac{\theta(2,2,12)}{\eta(z) \eta(23 z)}\right)=\left(\frac{\eta(z) \eta(23 z)}{\eta(2 z) \eta(46 z)}\right)+4\left(\frac{\eta(2 z) \eta(46 z)}{\eta(z) \eta(23 z)}\right)+4\left(\frac{\eta(2 z) \eta(46 z)}{\eta(z) \eta(23 z)}\right)^{2}+c_{1}$ \\
\hline 26 & $t_{26}=\left(\frac{\eta(2 z) \eta(13 z)}{\eta(z) \eta(26 z)}\right)^{2}+\left(\frac{\eta(z) \eta(26 z)}{\eta(2 z) \eta(13 z)}\right)^{2}$ \\
\hline 29 & $t_{29}=\frac{\theta_{x}\left(\frac{1}{2}, 0, \frac{29}{2}\right)-\theta_{y}\left(\frac{1}{2}, 0, \frac{29}{2}\right)}{2 \eta(z) \eta(29 z)}$ \\
\hline \multirow[t]{2}{*}{30} & $t_{30}=\left(\frac{\eta(z) \eta(6 z) \eta(10 z) \eta(15 z)}{\eta(2 z) \eta(3 z) \eta(5 z) \eta(30 z)}\right)^{3}+\left(\frac{\eta(z) \eta(6 z) \eta(10 z) \eta(15 z)}{\eta(2 z) \eta(3 z) \eta(5 z) \eta(30 z)}\right)^{-3}$ \\
\hline & $=\left(\frac{\eta(z) \eta(3 z) \eta(5 z) \eta(15 z)}{\eta(2 z) \eta(6 z) \eta(10 z) \eta(30 z)}\right)+4\left(\frac{\eta(z) \eta(3 z) \eta(5 z) \eta(15 z)}{\eta(2 z) \eta(6 z) \eta(10 z) \eta(30 z)}\right)^{-1}+c_{1}$ \\
\hline \multirow[t]{2}{*}{30} & $t_{30}=\left(\frac{\eta(3 z) \eta(5 z) \eta(6 z) \eta(10 z)}{\eta(z) \eta(2 z) \eta(15 z) \eta(30 z)}\right)+\left(\frac{\eta(3 z) \eta(5 z) \eta(6 z) \eta(10 z)}{\eta(z) \eta(2 z) \eta(15 z) \eta(30 z)}\right)^{-1}+c_{2}$ \\
\hline & $=\left(\frac{\eta(2 z) \eta(3 z) \eta(10 z) \eta(15 z)}{\eta(z) \eta(5 z) \eta(6 z) \eta(30 z)}\right)^{2}+\left(\frac{\eta(2 z) \eta(3 z) \eta(10 z) \eta(15 z)}{\eta(z) \eta(5 z) \eta(6 z) \eta(30 z)}\right)^{-2}+c_{3}$ \\
\hline 31 & $t_{31}=\left(\frac{\theta(2,2,16)-\theta(4,2,8)}{2 \eta(z) \eta(31 z)}\right)^{3}$ \\
\hline 33 & $t_{33}=\left(\frac{\eta(z) \eta(11 z)}{\eta(3 z) \eta(33 z)}\right)+3\left(\frac{\eta(z) \eta(11 z)}{\eta(3 z) \eta(33 z)}\right)^{-1}$ \\
\hline 34 & $t_{34}$ is deduced from the formula $t_{34}^{2}(z)+t_{34}(z)-6=j_{17}(z)+j_{17}(2 z)$ \\
\hline 35 & $t_{35}=\left(\frac{\eta(5 z) \eta(7 z)}{\eta(z) \eta(35 z)}\right)-\left(\frac{\eta(5 z) \eta(7 z)}{\eta(z) \eta(35 z)}\right)^{-1}$ \\
\hline 38 & $t_{38}$ is deduced from the formula $t_{38}^{2}(z)+t_{38}(z)-4=j_{19}(z)+j_{19}(2 z)$ \\
\hline 39 & $t_{39}=\left(\frac{\eta(3 z) \eta(13 z)}{\eta(z) \eta(39 z)}\right)+\left(\frac{\eta(3 z) \eta(13 z)}{\eta(z) \eta(39 z)}\right)^{-1}$ \\
\hline 41 & $t_{41}=\frac{\theta_{x}\left(\frac{3}{2}, 2, \frac{15}{2}\right)-\theta_{y}\left(\frac{3}{2}, 2, \frac{15}{2}\right)}{2 \eta(z) \eta(41 z)}$ \\
\hline \multirow[t]{2}{*}{42} & $t_{42}=\left(\frac{\eta(z) \eta(6 z) \eta(14 z) \eta(21 z)}{\eta(2 z) \eta(3 z) \eta(7 z) \eta(42 z)}\right)^{2}+\left(\frac{\eta(z) \eta(6 z) \eta(14 z) \eta(21 z)}{\eta(2 z) \eta(3 z) \eta(7 z) \eta(42 z)}\right)^{-2}$ \\
\hline & $=\left(\frac{\eta(2 z) \eta(6 z) \eta(7 z) \eta(21 z)}{\eta(z) \eta(3 z) \eta(14 z) \eta(42 z)}\right)+\left(\frac{\eta(2 z) \eta(6 z) \eta(7 z) \eta(21 z)}{\eta(z) \eta(3 z) \eta(14 z) \eta(42 z)}\right)^{-1}+c_{1}$ \\
\hline
\end{tabular}


TABLE 3. (Continued.)

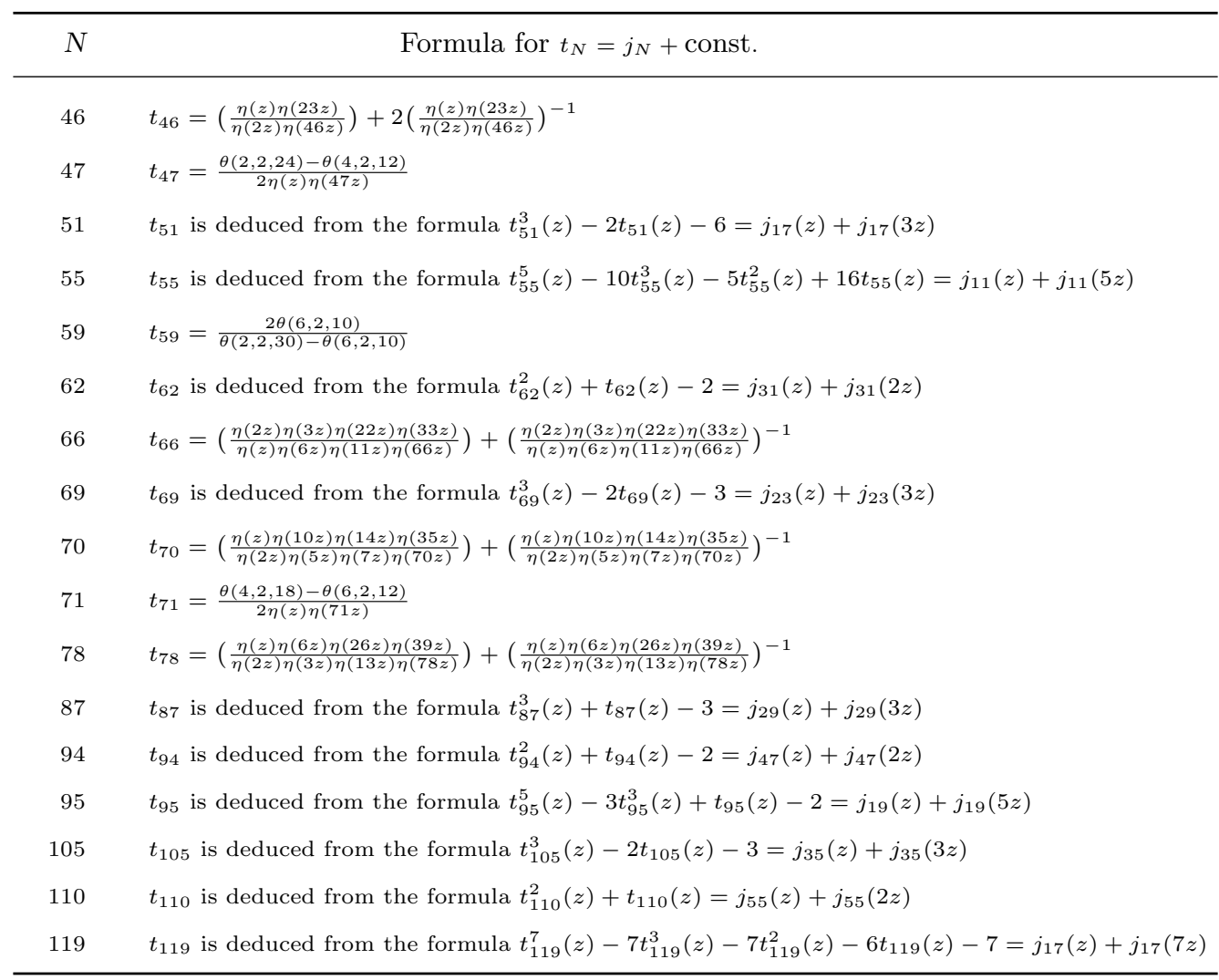

\subsection{Groups $\Gamma_{0}(N)^{+}$of higher genus}

There are 38 different square-free levels $N$ such that $X_{N}$ has genus one. Similarly, there are 39 and 31 different square-free $N$ such that $X_{N}$ has genus two and three, respectively. In [7], the authors studied the $q$-expansions for the corresponding function fields, proving that each function field admits two generators with various properties, such as minimal pole at infinity and integer coefficients. In particular, a polynomial relation was computed for each pair of generators, thus giving an algebraic equation for the corresponding projective curve. In future studies, we plan to investigate the various properties of these elliptic (genus-one) and hyperelliptic (genus-two) curves. There are a vast number of problems, both arithmetic and analytic, to be considered given that one knows the uniformizing group, a projective equation, $q$-expansions, and relations to holomorphic Eisenstein series.

\section{References}

1. A. Borel, S. Chowla, C. S. Herz, K. Iwasawa and J. P. Serre (eds), Seminar on complex multiplication, Lecture Notes in Mathematics 21 (Springer, Berlin-New York, 1966).

2. J. H. Conway and S. P. Norton, 'Monstrous moonshine', Bull. Lond. Math. Soc. 11 (1979) 308-339.

3. C. J. Cummins, 'Congruence subgroups of groups commensurable with $\operatorname{PSL}(2, \mathbb{Z})$ of genus 0 and 1', Exp. Math. 13 (2004) 361-382.

4. T. Gannon, 'Monstrous moonshine: the first twenty-five years', Bull. Lond. Math. Soc. 38 (2006) 1-33.

5. T. Gannon, Moonshine beyond the monster. The bridge connecting algebra, modular forms and physics, Cambridge Monographs on Mathematical Physics (Cambridge University Press, Cambridge, 2006). 
6. J. Jorgenson, L. Smajlović and H. Then, 'On the distribution of eigenvalues of Maass forms on certain moonshine groups', Math. Comp. 83 (2014) 3039-3070.

7. J. Jorgenson, L. Smajlović and H. Then, 'Kronecker's limit formula, holomorphic modular functions and $q$-expansions on certain arithmetic groups', Exp. Math. 25 (2016) 295-320.

8. J. Jorgenson, L. Smajlović and H. Then, Data page, http://www.efsa.unsa.ba/ lejla.smajlovic/.

9. T. Miezaki, H. Nozaki and J. Shigezumi, 'On the zeros of Eisenstein series for $\Gamma_{0}^{*}(2)$ and $\Gamma_{0}^{*}(3)$ ', J. Math. Soc. Japan 59 (2007) 693-706.

10. J.-P. SERre, A course in arithmetic, Graduate Texts in Mathematics 7 (Springer, New York, 1973).

Jay Jorgenson

Department of Mathematics The City College of New York Convent Avenue at 138th Street New York

NY 10031

USA

jjorgenson@mindspring.com

Holger Then

Department of Mathematics

University of Bristol

University Walk

Bristol, BS8 1TW

United Kingdom

holger.then@bristol.ac.uk
Lejla Smajlović

Department of Mathematics

University of Sarajevo

Zmaja od Bosne 35

71000 Sarajevo

Bosnia and Herzegovina

lejlas@pmf.unsa.ba 